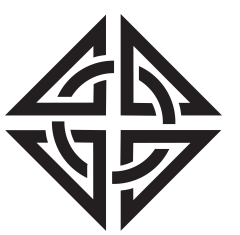

SCIENTIA
Sharif University of Technology

Scientia Iranica

Transactions F: Nanotechnology

www.scientiairanica.com

\title{
An analytical solution for bending, buckling, and free vibration of FG nanobeam lying on Winkler-Pasternak elastic foundation using different nonlocal higher order shear deformation beam theories
}

\author{
V. Refaeinejad, O. Rahmani* and S.A.H. Hosseini \\ Smart Structures and New Advanced Materials Laboratory, Department of Mechanical Engineering, University of Zanjan, Zanjan,
} Iran.

Received 24 December 2015; received in revised form 26 October 2016; accepted 19 December 2016

\author{
KEYWORDS \\ Winkler-Pasternak \\ elastic foundation; \\ Nonlocal Higher order \\ Shear Deformation \\ Theories (HSDTs); \\ Functionally Graded \\ (FG) nanobeam; \\ Bending; \\ Buckling; \\ Free vibration.
}

\begin{abstract}
In the present study, various Higher-order Shear Deformation beam Theories (HSDTs) are applied in order to achieve the exact analytical solution to bending, buckling, and free vibration of Functionally Graded (FG) nanobeam lying on the Winkler and Pasternak elastic foundations. HSDTs are those in which the effect of transverse shear strain is included. The displacement field of these theories involves a quadratic variation of transverse shear strains and stresses; hence, this hypothesis leads to the diminishing of transverse shear stresses at the top and bottom surfaces of a beam. Thus, necessarily, there is no need to use a shear correction factor in the HSDTs. Nanobeam has been made of FG materials in which the properties of these materials are changed through the thickness direction of nanobeam according to the power-law distribution. Hamilton's principle is used to derive the equation of motions and the related boundary conditions of simply supported nanobeam. The present study shows that the stability and vibration behaviors of FG nanobeam are extremely dependent on the Winkler and Pasternak elastic foundation, gradient index, aspect ratio, and nonlocal parameter. The obtained results of the present study might be useful in the advanced field of micro/nano electromechanical systems.
\end{abstract}

(C) 2017 Sharif University of Technology. All rights reserved.

\section{Introduction}

Nanotechnology is principally concerned with manufacturing of advanced materials at a nanoscale, which presents a novel class of structures with innovative properties and improved performance devices. The identification of mechanical behavior of nanostructures is critical in designing such structures for technological applications. Among these nanostructures

\footnotetext{
*. Corresponding author. Fax: +98 243228320 E-mail addresses: V.Refaeinejad@znu.ac.ir (V. Refaeinejad); omid.rahmani@znu.ac.ir (O.Rahmani); Hosseini@znu.ac.ir (S.A.H. Hosseini)
}

are nanobeams which attract increasing concentration owing to their numerous possible applications, such as nanoprobes, nanowires, Atomic Force Microscope (AFM), nanosensors and nanoactuators. Also, size effects are important on the mechanical performance of nanostructures in which sizes are small and analogous to molecular distances. This inspired many researchers to find a new model to predict the mechanical behavior of these nanostructures.

Sun and Zhang [1] examined the weaknesses of continuum models in the nanometer length scale. They observed that material properties were influenced by the length of plate structure. These results show that disconnected material structure at the nano-scale 
cannot be homogenized into a continuum. Currently, the nonlocal elastic continuum models are taken into account in the investigation of nanostructures. The nonlocal elasticity theory was first presented by Eringen [2-4]. This theory states that the stress field at a point of a body is a function of the strain field at every point of the continuum body.

Many studies used the nonlocal elasticity theory to take into account nanoscale influences on the nanostructures [5-13]. Civalek and Akgöz [7] used nonlocal Euler-Bernoulli theory and DQ method for static analysis of carbon nanotubes with various boundary conditions. Şimşek and Yurtcu [9] used nonlocal EulerBernoulli and Timoshenko beam model for bending and buckling evaluations of the FG nanobeam. Uymaz [10] used the nonlocal elasticity to analyze the free and forced vibration behavior of FG nanobeams. In this study, it is seen that the nonlocal parameter plays an important role in vibration modes. Torsional and axial vibration behavior of microtubules was investigated using nonlocal elasticity theory for both continuous and discrete modeling by Demir and Civalek [14]. It was concluded that the nonlocal effects play an important role in the static and buckling responses of the FG nanobeam.

A new generation of innovative inhomogeneous materials, constituted of two parts with dissimilar material properties and continuously changing composition spreading, has been established which is stated as Functionally Graded Materials (FGMs). Such materials have been presented to take benefit of the desired material properties of each component material without interface difficulties. Nowadays, FGMs have been massively applied into micro or nano elements such as micro-switches [15,16], micro-piezoactuator [17], micro/nano-electro mechanical systems (MEMS and NEMS) $[18,19]$, and thin films in the form of shape memory alloys $[20,21]$.

Beams resting on elastic foundations have extensive applications in engineering structures. This inspired many scientists to examine the performance of structures in different kinds of elastic foundations. The Winkler-type elastic foundation is estimated as a series of closely spaced, mutually independent, and vertical linear elastic springs. The foundation modulus is characterized by stiffness of the springs. However, this model is considered as a rough approximation of the real mechanical performance of the elastic material. This is due to failure of the model to consider the continuity or cohesion of the medium. The interaction among the springs is not taken into account in Winklertype foundations. A more accurate and generalized modeling of the elastic foundation can be achieved by the way of a two-parameter foundation model. One such physical foundation model is the Pasternak-type foundation model [22]. The first factor of Pasternak foundation model characterizes the normal pressure, while the second one accounts for the transverse shear stress caused by interaction of shear deformation of the surrounding elastic medium. Numerous researchers have studied the mechanical performance of nanoscale materials, particularly carbon nanotubes embedded in different surroundings, which can be modeled with various kinds of elastic foundations. Yoon and Midoduchowski studied the internal vibration of multi-wall carbon nanotubes surrounded by an elastic medium based on multiple-elastic beam model [23]. A combined method for bending and free vibration of arbitrary thick beams lying on Pasternak elastic foundation was presented by Chen et al. [24]. The influence of Winkler and Pasternak elastic foundations on bending and free vibration of $\mathrm{FG}$ beams was investigated by Ying et al. [25]. Murmu and Pradhan investigated a thermal vibration of single-carbon nanotube lying on single-elastic medium using thermal nonlocal elasticity theory [26]. Soltani et al. [27] proposed a Timoshenko elastic model to predict the vibrational behavior of a multi-wall carbon nanotube embedded in a Pasternak medium. They used a generalized differential quadrature method to solve the governing equations with different boundary conditions. The effect of elastic foundation on nonlinear vibration analysis and buckling of FG nanobeam has been presented recently by Niknam and Aghdam [28]. An EulerBernoulli nonlocal beam model was used to analyze the buckling behavior of protein microtubules lying on elastic foundation by Civalek and Demir [29]. Also, different boundary conditions were investigated using a finite element method. It may be concluded that the elastic foundation coefficients have direct relation with both natural frequency and buckling load.

Beams are the elementary structures largely used in MEMS, NEMS, and AFM with the order of microns or sub-microns, and their properties are thoroughly related to their microstructure. By the increase of using FGM in structures, many beam theories have been proposed to predict the performance of FG beams [30]. The Classical Beam Theory (CBT), identified as EulerBernoulli beam model, is the simplest one and is suitable just for slender FG beams. In place of moderately deep FG beams, the CBT overestimates natural frequency and underestimates deflection due to overlooking the transverse shear effect $[22,31,32]$. The Timoshenko beam model (First-order shear deformation Beam Theory-FBT) has been suggested to reduce the limitations of the CBT by taking into account the transverse shear influence. As the transverse shear strain and consequently stress are assumed to be constant through the thickness coordinate in FBT, a shear correction factor is necessary to explain the inconsistency between the supposed constant stress 
state and the real stress state [33-36]. To avoid the utilization of a shear correction factor and have an improved computation of FG beams behavior, HSDTs have been proposed. HSDTs can be set based on the assumption of a higher-order variation of axial displacement through the thickness direction of the beam [3739 ] or both axial and transverse displacements through the thickness of the beam [40-42].

Many investigations have been conducted trying to develop static and dynamic analyses of nanobeams based on various HSDTs. Thai and Vo focused on bending and free vibration behavior of FG beams using HSDTs in the microscopic scale [30]. Also, bending and vibration behavior of FG micro-beams based on the sinusoidal shear deformation theory was proposed by Lei et al. [43]. In addition, analytical solutions to bending and buckling of FG nanobeams via the nonlocal Timoshenko beam theory were presented by Şimşek and Yurtcu [9]. Besides, the Euler-Bernoulli beam theory was used to obtain static deflection and buckling response of FG nanobeams by Eltaher et al. [44]. Furthermore, the size effect on the vibrational behavior of FG nanobeams was studied based on nonlocal Timoshenko beam theory by Rahmani and Pedram [45]. Recently, vibrational analysis of FG nanobeams using various HSDTs has been presented by Refaeinejad et al. [46].

With all due respect to the author's knowledge, the bending, buckling, and free transverse vibration behavior of FG nanobeams embedded in an elastic foundation, involved in both Winkler and Pasternak elastic foundations, have not been considered in the previous studies. So, the ultimate aim of this study is to cover these issues in the literature.

This paper is the first comprehensive study in which nonlocal elasticity theory has been implemented to investigate the bending, buckling, and free transverse vibration of FG nanobeam. The Winkler and Pasternak elastic foundation models have been used to simulate the substrate medium. Also, HSDTs have improved so as to predict the static and dynamic behaviors of nano-structures accurately. The suggested models accommodate quadratic variation of transverse shear strains (and hence stresses); consequently, a shear correction factor is not necessary. Hamilton's principle is applied to derive the equations of motion and the related BCs. The Navier solution is employed for simply supported BCs, and analytical solutions to the bending, buckling, and free transverse vibration are presented for FG nanobeams. In the following, the influence of gradient index, Winkler and Pasternak parameters, size-scale parameter, and aspect ratio on the bending, buckling, and free transverse vibration characteristics of FG nanobeams are examined, and achieved results are compared with those in available literature to validate the accuracy of the present solution.

\section{Material properties}

Figure 1 indicates an FG nanobeam embedded in Winkler and Pasternak elastic foundations which has length, $l$, thickness, $h$, and width, $b$. All material properties such as Young's modulus, E, mass density, $\rho$, and Poisson's ratio, $\nu$, are assumed to vary with thickness $z$ in accordance with the power law which is evaluated as follows:

$$
\prod(Z)=\left(\prod_{1}-\prod_{2}\right)\left(\frac{z}{h}+\frac{1}{2}\right)^{p}+\prod_{2}
$$

$\Pi_{1}$ and $\Pi_{2}$ indicate the material properties at the upper and lower surfaces of the beam, respectively. The variation profile of material properties across the thickness of the beam is denoted by $p$. Based on distribution function, when $p=0$, the material is homogenous and is assumed to be an isotropic beam with bulk properties of the upper surfaces.

\section{Nonlocal beam theory}

Based on Eringen nonlocal theory, the stress field at point $X$ not only depends on the strain field of the same point, but also depends on strains at all points of the body. The aforementioned fact is proven by the

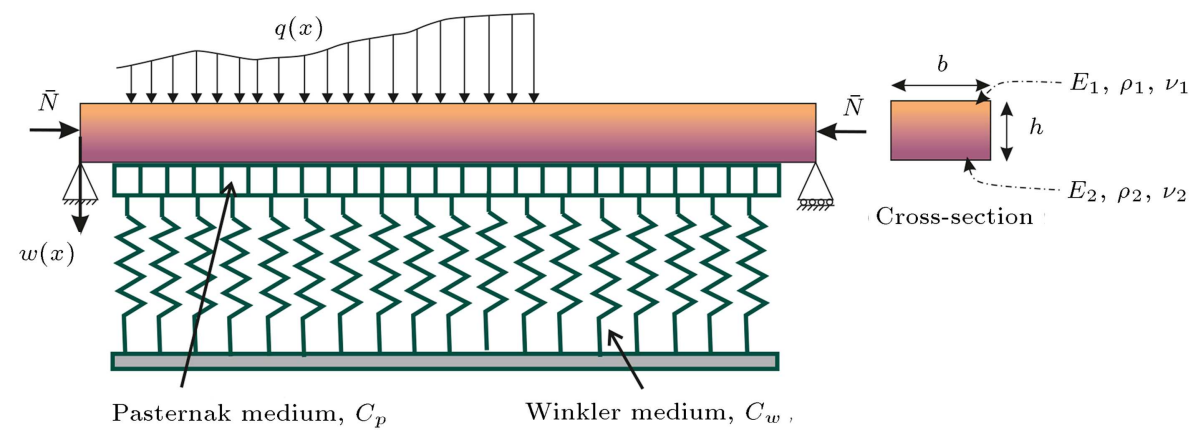

Figure 1. Schematic of a FG nanobeam lying on Winkler-Pasternak elastic foundation model under a transversed distributed load and an axial load. 
atomic theory of lattice dynamics and experimental observation of phonon dispersion. Stress tensor $\sigma$ at point $X$ is calculated as follows [2]:

$$
\bar{\sigma}=\int_{\Omega} K\left(\left|X^{\prime}-X, \tau\right|\right) \sigma\left(X^{\prime}\right) d X^{\prime}
$$

where $\sigma$ represents the classical, microscopic second Piola-Kirchhoff stress tensor at point $X$, kernel function $K\left|\left(X^{\prime}-X\right), \tau\right|$ denotes the nonlocal modulus, $\left(X^{\prime}-X\right)$ is the distance, and $\tau$ stands for material parameter which depends on internal and external characteristic lengths. Based on the generalized Hooke's law, macroscopic stress, $\sigma$, at point $X$ in Hookean solid is related to strain $\varepsilon$ at point $X$ which is as follows:

$$
\sigma(X)=C(X): \varepsilon(X) .
$$

The fourth-order elasticity tensor which denotes double-dot product is represented by $C$. Eqs. (2) and (3) together represent the nonlocal constitutive behavior of Hookean solid. The weighted average of the contributions of the strain field of all points in the body to the stress field at point $X$ is indicated by Eq. (2). For the sake of simplicity, an equivalent differential model is used instead of integral constitutive relation, which is evaluated as follows [2]:

$$
\left(1-\mu_{0}^{2} \nabla^{2}\right) \bar{\sigma}=\sigma, \quad \mu_{0}=\tau^{2} \ell^{2}=e_{0}^{2} a^{2},
$$

where $\sigma$ is the classical stress tensor at point $x\left(\sigma_{i j}=\right.$ $\left.C_{i j k l} \varepsilon_{k l}\right)$, and $\nabla^{2}$ is the Laplacian operator. For a beam type structure, the nonlocal behavior can be ignored in the thickness direction. Therefore, the nonlocal constitutive relation takes the following form [3]:

$$
\begin{gathered}
\sigma_{x x}-\mu \frac{\partial^{2} \sigma_{x x}}{\partial x^{2}}=E \varepsilon_{x x}, \\
\sigma_{x z}-\mu \frac{\partial^{2} \sigma_{x z}}{\partial x^{2}}=G \gamma_{x z} .
\end{gathered}
$$

$E$ and $G$ are elastic and shear moduli of the beam, respectively. The nonlocal parameter is denoted by $\mu=\left(e_{0} \alpha\right)^{2}$, and $\sigma_{x x}$ and $\sigma_{x z}$ represent the normal and tangential nonlocal stresses, sequentially.

\section{The governing equations}

Figure 1 shows that the rectangular Cartesian coordinate systems, $x, y, z$, are assumed to be the length, width, and height of the nanobeam, respectively. Based on HSDTs, the displacement field states that:

$$
\begin{aligned}
& u_{x}(x, z, t)=u(x, t)-z \frac{d w_{b}}{d x}-f(z) \frac{d w_{s}}{d x}, \\
& u_{z}(x, z, t)=w_{b}(x, t)+w_{s}(x, t),
\end{aligned}
$$

where $u, w_{b}$ and, $w_{s}$ are the axial displacement, bending and shear components of transverse displacement on the neutral axis of the nanobeam; $f(z)$ determines the transverse shear strain and shear stress distributions through the thickness direction of the nanobeam that is called a shape function. The different shape functions, $f(z)$, used in this study are listed in Table 1, such as Third-order Beam Theory of Reddy (TBT) [47], the Sinusoidal Beam Theory of Touratier (SBT) [48], the Hyperbolic Beam Theory of Soldatos (HBT) [49], the Exponential Beam Theory of Karama (EBT) [50], the Aydogdu (generalized) Beam Theory (ABT) [6]; and the formulations were proposed by Mantari et al. (HSDT1-HSDT3) [5153].

The non-zero strain fields are given as follows:

$$
\begin{aligned}
\varepsilon_{x} & =\frac{d u}{d x}-z \frac{d^{2} w_{b}}{d x^{2}}-f(z) \frac{d^{2} w_{s}}{d x^{2}}, \\
\gamma_{x z} & =g(z) \frac{d w_{s}}{d x},
\end{aligned}
$$

where $g(z)=1-d f / d z$ is the shape function of transverse shear strains. Through the depth of the nanobeam, the distribution of the transverse shear strain and the transverse shear stresses are represented by these shape functions.

Hamilton's principle defines that:

$$
0=\int_{t_{1}}^{t_{2}} \delta(U+V-T) d t,
$$

Table 1. Different higher-order shear deformation theories of shape functions used in this study.

\begin{tabular}{llcl}
\hline \multicolumn{1}{c}{ Model } & $\boldsymbol{f ( z )}$ & Abbreviation & Full form of theories \\
\hline Reddy [47] & $\frac{4 z^{3}}{3 h^{2}}$ & TBT & Third-order Beam Theory \\
Touratier[48] & $z-\frac{h}{\pi} \sin \left(\frac{\pi z}{h}\right)$ & SBT & Sinusoidal Beam Theory \\
Soldates [49] & $z-h \sinh \left(\frac{z}{\pi}\right)+z \cosh \left(\frac{1}{2}\right)$ & HBT & Hyperbolic Beam Theory \\
Karama et al. [50] & $z-z e^{-2(z / h)^{2}}$ & EBT & Exponential Beam Theory \\
Aydogdu [6] & $z-z \times 3^{\frac{-2}{\ln (3)} \times\left(\frac{z}{h}\right)^{2}}$ & ABT & Aydogdu Beam Theory \\
Mantari and Guedes Soares [51] $z-\tan (m z)+z m \sec ^{2}\left(\frac{m h}{2}\right)$ & HSDT1 & 1st Mantari Beam Theory \\
Mantari et al. [52] & $z-\sin \left(\frac{\pi z}{h}\right) e^{m \cos \left(\frac{\pi z}{h}\right)}-z \frac{m \pi}{h}$ & HSDT2 & 2nd Mantari Beam Theory \\
Mantari et al. [53] & $z-\tan \left(\frac{\pi z}{2 h}\right) m^{\sec \left(\frac{\pi z}{2 h}\right)}+z \frac{\pi m}{h \sqrt{2}}(\sqrt{2}+\ln (m))$ & HSDT3 & 3rd Mantari Beam Theory \\
Euler-Bernoulli (CBT) & $z$ & CBT & Classical Beam Theory \\
\hline
\end{tabular}


where virtual strain energy, $\delta U$, virtual kinetic energy, $\delta T$, and virtual potential of external loading, $\delta V$, are:

$$
\begin{aligned}
\delta U & =\int_{v}\left(\sigma_{x x} \delta \varepsilon_{x x}+\sigma_{x z} \delta \gamma_{x z}\right) d V \\
& =\int_{0}^{L}\left(N \frac{d \delta u}{d x}-M_{b} \frac{d^{2} \delta w_{b}}{d x^{2}}-M_{s} \frac{d^{2} \delta w_{s}}{d x^{2}}+Q \frac{d \delta w_{s}}{d x}\right) d x
\end{aligned}
$$

where $N, M_{b}, M_{s}$, and $Q$ are the stress resultants defined as follows:

$$
\left\{\begin{array}{c}
N \\
M_{b} \\
M_{z}
\end{array}\right\}=\int_{A}\left\{\begin{array}{l}
1 \\
z \\
f
\end{array}\right\} \sigma_{x x} d A, \quad Q=\int_{A} g \sigma_{x z} d A .
$$

The variation of the potential energy can be expressed as follows:

$$
\begin{aligned}
\delta V= & -\int_{0}^{L} q \delta\left(w_{b}+w_{s}\right) d x \\
& -\int_{0}^{L} \bar{N} \frac{d\left(w_{b}+w_{s}\right)}{d x} \frac{d \delta\left(w_{b}+w_{s}\right)}{d x} d x,
\end{aligned}
$$

where $q$ and $\bar{N}$ are the transverse and axial loads, respectively. In addition, the total transverse load, $q$, made of two parameters, is in the following form:

$$
q(x)=q_{0}+f(x) .
$$

$q_{0}$ represents the transverse distributed load form. In the present study, $f(x)$ caused by elastic foundation is considered as in the following form based on the Winkler and Pasternak foundations [54-57]:

$$
f(x)=-C_{w} W(x, t)+C_{p} \frac{\partial^{2} W(x, t)}{\partial x^{2}},
$$

where $C_{w}\left(\mathrm{nN} / \mathrm{nm}^{2}\right)$ and $C_{p}(\mathrm{nN})$ are the Winkler and Pasternak stiffness parameters of the elastic medium. follows:

The variation of kinetic energy is expressed as

$$
\begin{aligned}
\delta T= & \int_{0}^{L} \int_{A} \rho(z)\left(\frac{\partial u_{1}}{\partial t} \frac{\partial \delta u_{1}}{\partial t}+\frac{\partial u_{2}}{\partial t} \frac{\partial \delta u_{2}}{\partial t}+\frac{\partial u_{3}}{\partial t} \frac{\partial \delta u_{3}}{\partial t}\right) d A d x \\
= & \int_{0}^{L}\left\{I_{0}\left[\frac{\partial u}{\partial t} \frac{\partial \delta u}{\partial t}+\left(\frac{\partial w_{b}}{\partial t}+\frac{\partial w_{s}}{\partial t}\right) \delta\left(\frac{\partial w_{b}}{\partial t}+\frac{\partial w_{s}}{\partial t}\right)\right]\right. \\
& -I_{1}\left(\frac{\partial u}{\partial t} \frac{\partial^{2} \delta w_{b}}{\partial x \partial t}+\frac{\partial^{2} \delta w_{b}}{\partial x \partial t} \frac{\partial u}{\partial t}\right)
\end{aligned}
$$

$$
\begin{aligned}
& +I_{2} \frac{\partial^{2} w_{b}}{\partial x \partial t} \frac{\partial^{2} \delta w_{b}}{\partial x \partial t} \\
& -J_{1}\left(\frac{\partial u}{\partial t} \frac{\partial^{2} \delta w_{s}}{\partial x \partial t}+\frac{\partial^{2} \delta w_{s}}{\partial x \partial t} \frac{\partial u}{\partial t}\right) \\
& +k_{2} \frac{\partial^{2} w_{s}}{\partial x \partial t} \frac{\partial^{2} \delta w_{s}}{\partial x \partial t} \\
& \left.+J_{2}\left(\frac{\partial^{2} w_{b}}{\partial x \partial t} \frac{\partial^{2} w_{s}}{\partial x \partial t}+\frac{\partial^{2} w_{s}}{\partial x \partial t} \frac{\partial^{2} w_{b}}{\partial x \partial t}\right)\right\} d x,
\end{aligned}
$$

where $\rho$ is the mass density; $\left(I_{0}, I_{1}, J_{1}, I_{2}, J_{2}, k_{2}\right)$ are the mas inertias defined as follows:

$$
\left\{\begin{array}{l}
I_{0} \\
I_{1} \\
J_{1} \\
I_{2} \\
J_{2} \\
k_{2}
\end{array}\right\}=\int_{A}\left\{\begin{array}{c}
1 \\
z \\
f \\
z^{2} \\
z f \\
f^{2}
\end{array}\right\} \rho(z) d A
$$

where $\rho$ is the mass density in the form of power-law distribution.

By putting expressions $\delta U, \delta V$, and $\delta T$ from Eqs. (9), (11), and (13) in Eq. (8) and integrating them part by part and gathering the coefficients of $\delta u$, $\delta w_{b}$, and $\delta w_{s}$, the following equations of motion of the functionally-graded nanobeam are written as follows:

$$
\begin{aligned}
& \delta u: \quad \frac{d N}{d x}=I_{0} \frac{\partial^{2} u}{\partial t^{2}}-I_{1} \frac{\partial^{3} w_{b}}{\partial x \partial^{2} t}-J_{1} \frac{\partial^{3} w_{s}}{\partial x \partial t^{2}}, \\
& \delta w_{b}: \quad \frac{d^{2} M_{b}}{d x^{2}}-\bar{N}\left(\frac{d^{2} w_{b}}{d x^{2}}+\frac{d^{2} w_{s}}{d x^{2}}\right)+q_{0} \\
& -C_{w} W(x, t)+C_{p} \frac{\partial^{2} W(x, t)}{\partial x^{2}} \\
& =I_{0}\left(\frac{\partial^{2} w_{b}}{\partial t^{2}}+\frac{\partial^{2} w_{s}}{\partial t^{2}}\right)+I_{1} \frac{\partial^{3} u}{\partial x \partial^{2} t} \\
& -I_{2} \frac{\partial^{4} w_{b}}{\partial x^{2} \partial t^{2}}-J_{2} \frac{\partial^{4} w_{s}}{\partial x^{2} \partial t^{2}}, \\
& \delta w_{s}: \quad \frac{d^{2} M_{s}}{d x^{2}}-\bar{N}\left(\frac{d^{2} w_{b}}{d x^{2}}+\frac{d^{2} w_{s}}{d x^{2}}\right)+q_{0} \\
& -C_{w} W(x, t)+C_{p} \frac{\partial^{2} W(x, t)}{\partial x^{2}} \\
& =I_{0}\left(\frac{\partial^{2} w_{b}}{\partial t^{2}}+\frac{\partial^{2} w_{s}}{\partial t^{2}}\right)+J_{1} \frac{\partial^{3} u}{\partial x \partial t^{2}} \\
& -J_{2} \frac{\partial^{4} w_{b}}{\partial x^{2} \partial t^{2}}-k_{2} \frac{\partial^{4} w_{s}}{\partial x^{2} \partial t^{2}} .
\end{aligned}
$$


The boundary conditions are given as below in the specific form:

$$
\begin{aligned}
& u \text { or } N \text {, } \\
& w_{b} \quad \text { or } \quad Q_{b} \equiv \frac{d M_{b}}{d x^{2}}-\bar{N} \frac{d}{d x}\left(w_{b}+w_{s}\right)-I_{1} \frac{\partial^{2} u}{\partial t^{2}} \\
& +I_{2} \frac{\partial^{3} w_{b}}{\partial x \partial t^{2}}+J_{2} \frac{\partial^{3} w_{s}}{\partial x \partial t^{2}} \\
& w_{s} \quad \text { or } \quad Q_{s} \equiv \frac{d M_{s}}{d x}-\bar{N} \frac{d}{d x}\left(w_{b}+w_{s}\right)+Q-J_{1} \frac{\partial^{2} u}{\partial t^{2}} \\
& +J_{2} \frac{\partial^{3} w_{b}}{\partial x \partial t^{2}}+k_{2} \frac{\partial^{3} w_{s}}{\partial x \partial t^{2}} \\
& \frac{d w_{b}}{d x} \text { or } M_{b} \\
& \frac{d w_{s}}{d x} \text { or } M_{s} \text {. }
\end{aligned}
$$

Substituting Eq. (7) into Eq. (5) and the subsequent results in Eq. (10), the stress resultants are obtained as follows:

$$
\begin{aligned}
& N-\mu \frac{d^{2} N}{d x^{2}}=A \frac{d u}{d x}-B \frac{d^{2} w_{b}}{d x^{2}}-B_{s} \frac{d^{2} w_{s}}{d x^{2}} \\
& M_{b}-\mu \frac{d^{2} M_{b}}{d x^{2}}=B \frac{d u}{d x}-D \frac{d^{2} w_{b}}{d x^{2}}-D_{s} \frac{d^{2} w_{s}}{d x^{2}} \\
& M_{s}-\mu \frac{d^{2} M_{s}}{d x^{2}}=B_{s} \frac{d u}{d x}-D_{s} \frac{d^{2} w_{b}}{d x^{2}}-H_{s} \frac{d^{2} w_{s}}{d x^{2}}, \\
& Q-\mu \frac{d^{2} Q}{d x^{2}}=A_{s} \frac{d w_{s}}{d x}
\end{aligned}
$$

where:

$$
\begin{aligned}
& {\left[A, B, B_{s}, D, D_{s}, H_{s}\right]=\int_{A}\left(1, z, f, z^{2}, z f, f^{2}\right) Q_{11}(z) d A} \\
& A_{s}=\int_{A} g^{2} Q_{55}(z) d A .
\end{aligned}
$$

The reduced elastic constants are defined as follows [10]:

$$
\begin{aligned}
Q_{11}(z) & =\frac{E(z)}{1-\nu^{2}}, \\
Q_{55}(z) & =\frac{E(z)}{2(1+\nu)},
\end{aligned}
$$

where $E$ is the elasticity modulus and is defined as a function of thickness direction according to power-law distribution.

\section{Equations of motion in terms of displacements}

Substituting Eq. (17) into Eq. (15), the equations of motion can be written as follows:

$$
\begin{aligned}
& A \frac{\partial^{2} u}{\partial x^{2}}-B \frac{\partial^{3} w_{b}}{\partial x^{3}}-B_{s} \frac{\partial^{3} w_{s}}{\partial x^{3}}=I_{0}\left(\frac{\partial^{2} u}{\partial t^{2}}-\mu \frac{\partial^{4} u}{\partial x^{2} \partial t^{2}}\right) \\
& -I_{1}\left(\frac{\partial^{3} w_{b}}{\partial x \partial t^{2}}-\mu \frac{\partial^{5} w_{b}}{\partial t^{2} \partial x^{3}}\right) \\
& -J_{1}\left(\frac{\partial^{3} w_{s}}{\partial x \partial t^{2}}-\mu \frac{\partial^{5} w_{s}}{\partial t^{2} \partial x^{3}}\right), \\
& B \frac{\partial^{3} u}{\partial x^{3}}-D \frac{\partial^{4} w_{b}}{\partial x^{4}}-D_{s} \frac{\partial^{4} w_{s}}{\partial x^{4}}-\bar{N}\left(\frac{\partial^{2} w_{b}}{\partial x^{2}}+\frac{\partial^{2} w_{s}}{\partial x^{2}}\right) \\
& +\bar{N} \mu\left(\frac{\partial^{4} w_{b}}{\partial x^{4}}+\frac{\partial^{4} w_{s}}{\partial x^{4}}\right)+q_{0}-C_{w}\left(w_{b}+w_{s}\right) \\
& +C_{p} \frac{\partial^{2}\left(w_{b}+w_{s}\right)}{\partial x^{2}} \\
& -\mu\left(\frac{\partial^{2} q_{0}}{\partial x^{2}}-C_{w} \frac{\partial^{2}\left(w_{b}+w_{s}\right)}{\partial x^{2}}+C_{p} \frac{\partial^{4}\left(w_{b}+w_{s}\right)}{\partial x^{4}}\right) \\
& =I_{0}\left[\left(\frac{\partial^{2} w_{b}}{\partial t^{2}}+\frac{\partial^{2} w_{s}}{\partial t^{2}}\right)-\mu \frac{\partial^{4}\left(w_{b}+w_{s}\right)}{\partial x^{2} \partial t^{2}}\right] \\
& +I_{1}\left(\frac{\partial^{3} u}{\partial x \partial t^{2}}-\mu \frac{\partial^{5} u}{\partial x^{3} \partial t^{2}}\right) \\
& -I_{2}\left(\frac{\partial^{4} w_{b}}{\partial x^{2} \partial t^{2}}-\mu \frac{\partial^{6} w_{b}}{\partial x^{4} \partial t^{2}}\right) \\
& -J_{2}\left(\frac{\partial^{4} w_{s}}{\partial x^{2} \partial t^{2}}-\mu \frac{\partial^{6} w_{s}}{\partial x^{4} \partial t^{2}}\right) \\
& B_{s} \frac{\partial^{3} u}{\partial x^{3}}-D_{s} \frac{\partial^{4} w_{b}}{\partial x^{4}}-H_{s} \frac{\partial^{4} w_{s}}{\partial x^{4}}+A_{s} \frac{\partial^{2} w_{s}}{\partial x^{2}} \\
& -\bar{N}\left(\frac{\partial^{2} w_{b}}{\partial x^{2}}+\frac{\partial^{2} w_{s}}{\partial x^{2}}\right)+\bar{N} \mu\left(\frac{\partial^{4} w_{b}}{\partial x^{4}}+\frac{\partial^{4} w_{s}}{\partial x^{4}}\right) \\
& +q_{0}-C_{w}\left(w_{b}+w_{s}\right)+C_{p} \frac{\partial^{2}\left(w_{b}+w_{s}\right)}{\partial x^{2}} \\
& -\mu\left(\frac{\partial^{2} q_{0}}{\partial x^{2}}-C_{w} \frac{\partial^{2}\left(w_{b}+w_{s}\right)}{\partial x^{2}}+C_{p} \frac{\partial^{4}\left(w_{b}+w_{s}\right)}{\partial x^{4}}\right) \\
& =I_{0}\left[\left(\frac{\partial^{2} w_{b}}{\partial t^{2}}+\frac{\partial^{2} w_{s}}{\partial t^{2}}\right)-\mu \frac{\partial^{4}\left(w_{b}+w_{s}\right)}{\partial x^{2} \partial t^{2}}\right] \\
& +J_{1}\left(\frac{\partial^{3} u}{\partial x \partial t^{2}}-\mu \frac{\partial^{5} u}{\partial x^{3} \partial t^{2}}\right) \\
& -J_{2}\left(\frac{\partial^{4} w_{b}}{\partial x^{2} \partial t^{2}}-\mu \frac{\partial^{6} w_{b}}{\partial x^{4} \partial t^{2}}\right) \\
& -K_{2}\left(\frac{\partial^{4} w_{s}}{\partial x^{2} \partial t^{2}}-\mu \frac{\partial^{6} w_{s}}{\partial x^{4} \partial t^{2}}\right)
\end{aligned}
$$




\section{Analytical solutions}

In this section, the governing equations are analytically solved for bending, buckling, and free vibration of a simply-supported FG nanobeam lying on Winkler and Pasternak elastic foundations. The Navier solution procedure is used to determine the analytical solutions to simply-supported boundary conditions.

The solution is assumed to be of the following form:

$$
\begin{aligned}
& u(x, t)=\sum_{n=1}^{\infty} U_{n} e^{i \omega t} \cos \alpha x, \\
& w_{b}(x, t)=\sum_{n=1}^{\infty} w_{b n} e^{i \omega t} \sin \alpha x, \\
& w_{s}(x, t)=\sum_{n=1}^{\infty} w_{s n} e^{i \omega t} \sin \alpha x,
\end{aligned}
$$

where $\left(U_{n}, W_{b n}\right.$, and $\left.W_{s n}\right)$ are the unknown displacement coefficients, $\omega$ is the vibration frequency, $\alpha=\frac{n \pi}{L}$, and $i=\sqrt{-1}$. The applied transverse, $q_{0}$, is expanded in Fourier series as follows:

$$
\begin{aligned}
& q_{0}(x)=\sum_{n=1}^{\infty} Q_{n} \sin \frac{n \pi}{L} x, \\
& Q_{n}=\frac{2}{L} \int_{0}^{L} q_{0}(x) \sin \frac{n \pi}{L} x d x,
\end{aligned}
$$

where $Q_{n}$ is the Fourier coefficient.

Substituting the expansions of $u, w_{b}, w_{s}$, and $q$ from Eqs. (21) and (22) into Eq. (20), the analytical solutions can be obtained from the following equations:

$$
\begin{gathered}
\left(\left[S_{i j}\right]-\bar{N} \alpha^{2}\left(1+\mu \alpha^{2}\right)\left[\begin{array}{lll}
0 & 0 & 0 \\
0 & 1 & 1 \\
0 & 1 & 1
\end{array}\right]\right. \\
\left.+\omega^{2}\left(1+\mu \alpha^{2}\right)\left[M_{i j}\right]\right)\left\{\begin{array}{c}
U_{n} \\
W_{b n} \\
W_{s n}
\end{array}\right\} \\
=\left(1+\mu \alpha^{2}\right)\left\{\begin{array}{c}
0 \\
Q_{n} \\
Q_{n}
\end{array}\right\} .
\end{gathered}
$$

The expressions of $S_{i j}$ and $M_{i j}$ are given in the Appendix.

\subsection{Bending}

The static deflections are obtained from Eq. (23) by setting $\bar{N}$ and $\omega$ to zero:

$u(x)=\frac{Q_{n}\left(s_{13}\left(s_{22}-s_{23}\right)+s_{12}\left(-s_{23}+s_{33}\right)\right)\left(1+\alpha^{2} \mu\right)}{s_{13}^{2} s_{22}-2 s_{12} s_{13} s_{23}+s_{12}^{2} s_{33}+s_{11}\left(s_{23}^{2}-s_{22} s_{33}\right)}$,

$$
\begin{aligned}
& w_{b}(x)=\frac{Q_{n}\left(-s_{12} s_{13}+s_{13}^{2}+s_{11}\left(s_{23}-s_{33}\right)\right)\left(1+\alpha^{2} \mu\right)}{s_{13}^{2} s_{22}-2 s_{12} s_{13} s_{23}+s_{12}^{2} s_{33}+s_{11}\left(s_{23}^{2}-s_{22} s_{33}\right)}, \\
& w_{s}(x)=\frac{Q_{n}\left(s_{12}^{2}-s_{12} s_{13}+s_{11}\left(-s_{22}+s_{23}\right)\right)\left(1+\alpha^{2} \mu\right)}{s_{13}^{2} s_{22}-2 s_{12} s_{13} s_{23}+s_{12}^{2} s_{33}+s_{11}\left(s_{23}^{2}-s_{22} s_{33}\right)}
\end{aligned}
$$

\subsection{Buckling}

The buckling load is obtained from Eq. (23) by setting $q$ and $\omega$ to zero:

$\bar{N}=\frac{-\left(s_{13}^{2} s_{22}-2 s_{12} s_{13} s_{23}+s_{12}^{2} s_{33}+s_{11}\left(s_{23}^{2}-s_{22} s_{33}\right)\right)}{\left(\left(s_{12}-s_{13}\right)^{2}-s_{11}\left(s_{22}-2 s_{23}+s_{33}\right)\right) \alpha^{2}\left(1+\alpha^{2} \mu\right)}$.

\section{Numerical results and discussion}

In this section, an $\mathrm{Al} / \mathrm{Al}_{2} \mathrm{O}_{3}$ beam, formed of aluminum (as metal) and alumina (as ceramic), has been considered for numerical results. The material properties of aluminum are $E_{m}=70 \mathrm{GPa}, \nu_{m}=0.3$, and $\rho_{m}=2702 \mathrm{~kg} / \mathrm{m}^{3}$, and those of alumina are $E_{c}=380 \mathrm{GPa}, \nu_{c}=0.3$, and $\rho_{c}=3960 \mathrm{~kg} / \mathrm{m}^{3}$, where $E, \nu$, and $\rho$ are the young modulus, Poisson's ratio, and mass density, respectively. For convenience, the dimensionless form is used in the following formulations:

$$
\begin{aligned}
& \bar{\omega}=\frac{\omega L^{2}}{h} \sqrt{\frac{\rho_{m}\left(1-\nu_{m}^{2}\right)}{E_{m}}}, \\
& \bar{w}=100 \frac{E_{m} h^{3}}{q_{0} L^{4}\left(1-\nu_{m}^{2}\right)} w\left(\frac{L}{2}\right), \\
& \bar{N}=\frac{N_{c r} 12 L^{2}\left(1-\nu_{m}^{2}\right)}{E_{m} b h^{3}} .
\end{aligned}
$$

\subsection{Verification of new results of nanobeam}

In this section, the numerical results obtained from this work have been compared with a nanobeam without elastic foundation $\left(C_{w}, C_{p}=0\right)$. Variation of transverse displacement, dimensionless buckling loads, and fundamental frequency versus the nonlocal and gradient index parameters of nanobeam are proposed in Tables 2 and 3 for two different aspect ratios. It can be seen that the present results show good agreement with those of the previous literature $[30,46,58]$.

\subsection{Parametric results}

In this section, the bending, buckling, and free transverse vibration of FG nanobeam embedded in Winkler and Pasternak elastic foundations are studied parametrically for different aspect ratios, gradient index, nonlocal, and Winkler-Pasternak elastic stiffness parameters.

In Tables 4 and 5, the effects of the Pasternak and Winkler coefficients on the dimensionless fundamental frequency of the FG nanobeam with respect to different nonlocal parameter, gradient index, and two aspect ratios have been proposed. As the tables show, the 
Table 2. Comparison of dimensionless transverse deflection of FG nanobeam without elastic foundation between Thai [30] and the present study for different aspect ratios and gradient indexes.

\begin{tabular}{|c|c|c|c|c|c|c|}
\hline \multirow{2}{*}{$L / h$} & \multirow{2}{*}{$p$} & \multirow{2}{*}{ Method } & \multicolumn{4}{|c|}{ Theory } \\
\hline & & & TBT & SBT & HBT & EBT \\
\hline \multirow{10}{*}{5} & \multirow{2}{*}{0} & Thai [30] & 3.1654 & 3.1649 & 3.1654 & 3.1635 \\
\hline & & Present & 3.1938 & 3.1932 & 3.1937 & 3.1938 \\
\hline & \multirow{2}{*}{0.5} & Thai [30] & 4.8285 & 4.8278 & 4.8285 & 4.8260 \\
\hline & & Present & 4.8669 & 4.8662 & 4.8669 & 4.8670 \\
\hline & \multirow{2}{*}{1} & Thai [30] & 6.2594 & 6.2586 & 6.2594 & 6.2563 \\
\hline & & Present & 6.3074 & 6.3065 & 6.3073 & 6.3075 \\
\hline & \multirow{2}{*}{2} & Thai [30] & 8.0677 & 8.0683 & 8.0675 & 8.0667 \\
\hline & & Present & 8.1337 & 8.1343 & 8.1334 & 8.1335 \\
\hline & \multirow{2}{*}{5} & Thai[30] & 9.8281 & 9.8367 & 9.8271 & 9.8414 \\
\hline & & Present & 9.9345 & 9.9440 & 9.9333 & 9.9333 \\
\hline \multirow{10}{*}{20} & \multirow{2}{*}{0} & Thai [30] & 2.8962 & 2.8962 & 2.8962 & 2.8961 \\
\hline & & Present & 2.8981 & 2.8980 & 2.8980 & 2.8981 \\
\hline & \multirow{2}{*}{0.5} & Thai [30] & 4.4644 & 4.4644 & 4.4644 & 4.4643 \\
\hline & & Present & 4.4669 & 4.4668 & 4.4668 & 4.4670 \\
\hline & \multirow{2}{*}{1} & Thai [30] & 5.8049 & 5.8049 & 5.8049 & 5.8047 \\
\hline & & Present & 5.8080 & 5.8079 & 5.8079 & 5.8080 \\
\hline & \multirow{2}{*}{2} & Thai [30] & 7.4421 & 7.4421 & 7.4420 & 7.4420 \\
\hline & & Present & 7.4462 & 7.4463 & 7.4462 & 7.4462 \\
\hline & \multirow{2}{*}{5} & Thai [30] & 8.8182 & 8.8188 & 8.8181 & 8.8191 \\
\hline & & Present & 8.8249 & 8.8255 & 8.8248 & 8.8248 \\
\hline
\end{tabular}

dimensionless fundamental frequency decreases as the gradient index increases. It should be considered that as the gradient index decreases, the FG nanobeam becomes stiffer; that is, the value of the dimensionless fundamental frequency decreases. Also, the dimensionless fundamental frequency reduces by the increase in the value of nonlocal parameter, especially for lower values of the aspect ratio such as the effect of gradient index. So, it can be considered that the effect of the nonlocal parameter is more prominent for the lower values of aspect ratio. As a result, the influence of nonlocal parameter can be ignored for higher values of aspect ratios. This shows that, for slender nanobeam, the effect of stiffness of elastic foundation on fundamental frequency becomes more significant as compared to the nonlocal effect.

The effects of Pasternak and Winkler coefficients are the same on the dimensionless fundamental frequency of FG nanobeam. It should be noted that the dimensionless fundamental frequency obtained from the Winkler foundation model is relatively larger than those obtained from the Pasternak foundation model, especially for slender nanobeam. Also, it can be seen in Tables 4 and 5 that the effect of the increase on these elastic foundation coefficients leads to an increase in the dimensionless fundamental frequency, especially for higher value of aspect ratio $(L / h>20)$. Moreover, it can be observed that the divergence of dimensionless fundamental frequency between thick nanobeam $(L / h=5)$ and thin nanobeam $(L / h=20)$ becomes noticeable for the higher values of the elastic coefficients (i.e., $C_{p}, C_{w}>10^{10}$ ). Therefore, the influence of shear deformation can be disappeared for the lower value of the elastic coefficients $\left(C_{p}, C_{w}<\right.$ $\left.10^{8}\right)$.

The variations of dimensionless frequencies and buckling loads with different nonlocal parameters and foundation parameters are listed in Tables 6-9. Five different mode numbers and two aspect ratios have been taken into consideration. In general, dimensionless frequency values increase due to the increase in mode number. It is also seen that the effect of 
Table 3. Comparison of dimensionless fundamental frequency of FG nanobeam without elastic foundation between previous studies and the present study for different aspect ratios and gradient indexes.

\begin{tabular}{|c|c|c|c|c|c|c|c|c|}
\hline \multirow{2}{*}{$L / h$} & \multirow{2}{*}{ Theory } & \multirow{2}{*}{ Method } & \multicolumn{6}{|c|}{$p$} \\
\hline & & & $\mathbf{0}$ & 0.5 & 1 & 2 & 5 & 10 \\
\hline \multirow{17}{*}{5} & \multirow{4}{*}{ TBT } & Şimşek [58] & 5.1527 & 4.4111 & 3.9904 & 3.6264 & 3.4012 & 3.2816 \\
\hline & & Thai and Vo [30] & 5.1527 & 4.4107 & 3.9904 & 3.6264 & 3.4012 & 3.2816 \\
\hline & & Refaeinejad et al. [46] & 5.1528 & 4.4107 & 3.9905 & 3.6265 & 3.4012 & 3.2816 \\
\hline & & Present & 5.13046 & 4.39375 & 3.9757 & 3.6123 & 3.3837 & 3.2627 \\
\hline & \multirow{4}{*}{ SBT } & Şimșek [58] & 5.1531 & 4.4114 & 3.9907 & 3.6263 & 3.3998 & 3.2811 \\
\hline & & Thai and Vo [30] & 5.1531 & 4.4110 & 3.9907 & 3.6263 & 3.3998 & 3.2811 \\
\hline & & Refaeinejad et al. [46] & 5.1532 & 4.4111 & 3.9908 & 3.6263 & 3.9998 & 3.2811 \\
\hline & & Present & 5.1309 & 4.3941 & 3.9760 & 3.6122 & 3.3822 & 3.2622 \\
\hline & & & & & & & & \\
\hline & \multirow{4}{*}{ HBT } & Şimşek [58] & 5.1527 & 4.4111 & 3.9904 & 3.6265 & 3.4014 & 3.2817 \\
\hline & & Thai and Vo [30] & 5.1527 & 4.4107 & 3.9904 & 3.6265 & 3.4014 & 3.2817 \\
\hline & & Refaeinejad et al. [46] & 5.1229 & 4.4108 & 3.9904 & 3.6265 & 3.4014 & 3.2817 \\
\hline & & Present & 5.1305 & 4.3937 & 3.9757 & 3.6123 & 3.3838 & 3.2628 \\
\hline & \multirow{4}{*}{ EBT } & Şimşek [58] & 5.1542 & 4.4122 & 3.9914 & 3.6267 & 3.3991 & 3.2813 \\
\hline & & Thai and Vo [30] & 5.1542 & 4.4118 & 3.9914 & 3.6267 & 3.3991 & 3.2814 \\
\hline & & Refaeinejad et al. [46] & 5.1543 & 4.4119 & 3.9915 & 3.6267 & 3.3991 & 3.2814 \\
\hline & & Present & 5.1321 & 4.3950 & 3.9769 & 3.6126 & 3.3814 & 3.2625 \\
\hline \multirow{17}{*}{20} & \multirow{4}{*}{ TBT } & Şimşek [58] & 5.4603 & 4.6516 & 4.2050 & 3.8361 & 3.6485 & 3.5390 \\
\hline & & Thai and Vo [30] & 5.4603 & 4.6511 & 4.2051 & 3.8361 & 3.6485 & 3.5390 \\
\hline & & Refaeinejad et al. [46] & 5.4604 & 4.6512 & 4.2050 & 3.8361 & 3.6485 & 3.5390 \\
\hline & & Present & 5.4586 & 4.6498 & 4.2039 & 3.8350 & 3.6471 & 3.5374 \\
\hline & \multirow{4}{*}{ SBT } & Şimşek [58] & 5.4603 & 4.6516 & 4.2050 & 3.8361 & 3.6485 & 3.5390 \\
\hline & & Thai and Vo [30] & 5.4603 & 4.6511 & 4.2051 & 3.8361 & 3.6485 & 3.5390 \\
\hline & & Refaeinejad et al. [46] & 5.4605 & 4.6512 & 4.2052 & 3.8361 & 3.6485 & 3.5390 \\
\hline & & Present & 5.4587 & 4.6499 & 4.2040 & 3.8351 & 3.6470 & 3.5390 \\
\hline & \multirow{5}{*}{ HBT } & & & & & & & \\
\hline & & Şimşek [58] & 5.4603 & 4.6516 & 4.2050 & 3.8361 & 3.6485 & 3.5390 \\
\hline & & Thai and Vo [30] & 5.4603 & 4.6511 & 4.2051 & 3.8361 & 3.6485 & 3.5390 \\
\hline & & Refaeinejad et al. [46] & 5.4605 & 4.6512 & 4.2951 & 3.8361 & 3.6485 & 3.5390 \\
\hline & & Present & 5.4586 & 4.6498 & 4.2039 & 3.8350 & 3.6471 & 3.5375 \\
\hline & \multirow{4}{*}{ EBT } & Şimşek [58] & 5.4604 & 4.6517 & 4.2052 & 3.8362 & 3.6483 & 3.5390 \\
\hline & & Thai and Vo [30] & 5.4604 & 4.6512 & 4.2051 & 3.8361 & 3.6483 & 3.5390 \\
\hline & & Refaeinejad et al. [46] & 5.4605 & 4.6512 & 4.2052 & 3.8361 & 3.6483 & 3.5390 \\
\hline & & Present & 5.4588 & 4.6500 & 4.2041 & 3.8351 & 3.6469 & 3.5375 \\
\hline
\end{tabular}

nonlocal parameter is more significant for higher mode numbers. Moreover, it can be observed that the effect of mode number on the increase in dimensionless frequency is more significant for thin nanobeam $(L / h>10)$. Finally, the effect of the increase in foundation parameters on the increase in dimensionless frequency is more significant for higher modes. It can be observed that the increase in foundation parameters and nonlocal parameter results in lower buckling loads. Also, it can be argued from these tables that, on one hand, the buckling load increases when the mode number increases, then it decreases due to the increase of mode numbers for thick nanobeams $(L / h<10)$. On the other hand, the buckling load decreases with the increase of mode number continuously for thin nanobeams $(L / h>10)$.

Figures 2-5 have been plotted in order to present the difference percentage of fundamental frequency between various HSDTs and Euler-Bernoulli Theory (CBT) with respect to the nonlocal parameter. Table 1 shows various HSDTs used in these figures. In other words, these figures have been drawn in order to represent the fundamental Frequency Difference Percentage Between various HSDTs and CBT (FDPBHC). Also, these fundamental frequencies have varied by the various nonlocal parameter, aspect ratios, and gradient indexes.

The formulation used in vertical axis is given by 
Table 4. The variation of dimensionless fundamental frequency of FG nanobeam lying on Pasternak foundation $\left(C_{w}=0\right)$ for different gradient indexes and nonlocal parameters.

\begin{tabular}{|c|c|c|c|c|c|c|c|c|c|}
\hline \multirow{3}{*}{$p$} & \multirow{3}{*}{$\mu\left(\mathrm{nm}^{2}\right)$} & \multicolumn{6}{|c|}{$C_{p}(\mathrm{nN})$} & \multirow{2}{*}{\multicolumn{2}{|c|}{$10^{10}$}} \\
\hline & & \multicolumn{2}{|c|}{$10^{4}$} & \multicolumn{2}{|c|}{$10^{6}$} & \multicolumn{2}{|c|}{$10^{8}$} & & \\
\hline & & $l / h=5$ & $l / h=20$ & $l / h=5$ & $l / h=20$ & $l / h=5$ & $l / h=20$ & $l / h=5$ & $l / h=20$ \\
\hline \multirow{4}{*}{0} & 0 & 5.13046 & 5.45864 & 5.130668 & 5.461807 & 5.151139 & 5.769846 & 6.901994 & 19.47484 \\
\hline & 1 & 4.34413 & 5.392518 & 4.344375 & 5.395725 & 4.368596 & 5.707331 & 6.33925 & 19.45641 \\
\hline & 2 & 3.835151 & 5.328742 & 3.835436 & 5.331988 & 3.862839 & 5.647112 & 6.00187 & 19.43883 \\
\hline & 3 & 3.471326 & 5.267178 & 3.47163 & 5.270461 & 3.501892 & 5.589055 & 5.77613 & 19.42204 \\
\hline \multirow{4}{*}{0.5} & 0 & 4.393753 & 4.64987 & 4.394022 & 4.654029 & 4.420771 & 5.052665 & 6.566526 & 20.30941 \\
\hline & 1 & 3.720337 & 4.593546 & 3.720654 & 4.597756 & 3.752206 & 5.000879 & 6.136283 & 20.29658 \\
\hline & 2 & 3.284443 & 4.53922 & 3.284802 & 4.54348 & 3.320499 & 4.951025 & 5.882133 & 20.28436 \\
\hline & 3 & 2.972861 & 4.486777 & 2.973258 & 4.491087 & 3.012648 & 4.902989 & 5.713902 & 20.27268 \\
\hline \multirow{4}{*}{1} & 0 & 3.975744 & 4.203993 & 3.976059 & 4.208881 & 4.007394 & 4.671955 & 6.408574 & 20.81039 \\
\hline & 1 & 3.366395 & 4.15307 & 3.366767 & 4.158018 & 3.403716 & 4.626186 & 6.049262 & 20.80016 \\
\hline & 2 & 2.971971 & 4.103954 & 2.972392 & 4.108961 & 3.014179 & 4.582144 & 5.838767 & 20.79041 \\
\hline & 3 & 2.690032 & 4.05654 & 2.690497 & 4.061606 & 2.736592 & 4.539727 & 5.700214 & 20.7811 \\
\hline \multirow{4}{*}{10} & 0 & 3.262751 & 3.537521 & 3.263205 & 3.544388 & 3.308255 & 4.174418 & 6.366162 & 22.4437 \\
\hline & 1 & 2.762681 & 3.494672 & 2.763216 & 3.501623 & 2.816276 & 4.138169 & 6.124184 & 22.43698 \\
\hline & 2 & 2.438992 & 3.453343 & 2.439598 & 3.460377 & 2.499537 & 4.103326 & 5.984322 & 22.43057 \\
\hline & 3 & 2.207615 & 3.413446 & 2.208285 & 3.420562 & 2.274328 & 4.069806 & 5.892926 & 22.42445 \\
\hline
\end{tabular}

Table 5. The variation of dimensionless fundamental frequency of FG nanobeam lying on Winkler foundation $\left(C_{p}=0\right)$ for different gradient indexes and nonlocal parameters.

\begin{tabular}{|c|c|c|c|c|c|c|c|c|c|}
\hline \multirow{3}{*}{$p$} & \multirow{3}{*}{$\mu\left(\mathrm{nm}^{2}\right)$} & \multicolumn{6}{|c|}{$C_{w}\left(\mathrm{nN} / \mathrm{nm}^{2}\right)$} & \multirow{2}{*}{\multicolumn{2}{|c|}{$10^{7}$}} \\
\hline & & \multicolumn{2}{|c|}{$10^{4}$} & \multicolumn{2}{|c|}{$10^{5}$} & \multicolumn{2}{|c|}{$10^{6}$} & & \\
\hline & & $l / h=5$ & $l / h=20$ & $l / h=5$ & $l / h=20$ & $l / h=5$ & $l / h=20$ & $l / h=5$ & $l / h=20$ \\
\hline \multirow{4}{*}{0} & 0 & 5.130466 & 5.459904 & 5.130513 & 5.471565 & 5.130987 & 5.586837 & 5.13572 & 6.630233 \\
\hline & 1 & 4.34413 & 5.393798 & 4.344192 & 5.405602 & 4.344752 & 5.522251 & 4.35034 & 6.575903 \\
\hline & 2 & 3.83515 & 5.330038 & 3.835219 & 5.341983 & 3.835852 & 5.459991 & 3.842182 & 6.523707 \\
\hline & 3 & 3.47133 & 5.268489 & 3.4714 & 5.280573 & 3.4721 & 5.399923 & 3.479091 & 6.473517 \\
\hline \multirow{4}{*}{0.5} & 0 & 4.39375 & 4.651531 & 4.393819 & 4.66683 & 4.394437 & 4.817153 & 4.400611 & 6.120583 \\
\hline & 1 & 3.72034 & 4.595227 & 3.720415 & 4.610713 & 3.721144 & 4.762807 & 3.728433 & 6.077903 \\
\hline & 2 & 3.28444 & 4.540921 & 3.284531 & 4.556592 & 3.285358 & 4.710434 & 3.293611 & 6.03695 \\
\hline & 3 & 2.97286 & 4.488499 & 2.972959 & 4.504352 & 2.973872 & 4.659919 & 2.982987 & 5.997617 \\
\hline \multirow{4}{*}{1} & 0 & 3.97574 & 4.205945 & 3.975822 & 4.22392 & 3.976546 & 4.399625 & 3.983783 & 5.874416 \\
\hline & 1 & 3.36640 & 4.155046 & 3.366486 & 4.17324 & 3.367342 & 4.350993 & 3.375885 & 5.838081 \\
\hline & 2 & 2.97197 & 4.105954 & 2.972075 & 4.124364 & 2.973044 & 4.304135 & 2.982716 & 5.803244 \\
\hline & 3 & 2.69004 & 4.058563 & 2.690147 & 4.077187 & 2.691217 & 4.258951 & 2.701899 & 5.769811 \\
\hline \multirow{4}{*}{10} & 0 & 3.26275 & 3.540264 & 3.262863 & 3.56548 & 3.263907 & 3.808465 & 3.274334 & 5.694027 \\
\hline & 1 & 2.76268 & 3.497449 & 2.762812 & 3.522971 & 2.764046 & 3.768697 & 2.77635 & 5.667506 \\
\hline & 2 & 2.43900 & 3.456153 & 2.439141 & 3.481978 & 2.440538 & 3.730405 & 2.454465 & 5.642115 \\
\hline & 3 & 2.20762 & 3.416289 & 2.20778 & 3.442413 & 2.209323 & 3.693503 & 2.224698 & 5.617785 \\
\hline
\end{tabular}


Table 6. Dimensionless frequency $\bar{\omega}$ of FG nanobeam with respect to increasing Pasternak coefficient, mode number, and nonlocal parameter.

\begin{tabular}{|c|c|c|c|c|c|c|c|}
\hline \multirow{3}{*}{$n$} & \multirow{3}{*}{$\mu\left(\mathrm{nm}^{2}\right)$} & \multicolumn{6}{|c|}{$C_{p}(\mathrm{nN})$} \\
\hline & & \multicolumn{2}{|c|}{$10^{4}$} & \multicolumn{2}{|c|}{$10^{6}$} & \multicolumn{2}{|c|}{$10^{8}$} \\
\hline & & $l / h=5$ & $l / h=20$ & $l / h=5$ & $l / h=20$ & $l / h=5$ & $l / h=20$ \\
\hline \multirow{3}{*}{1} & 0 & 3.9757 & 4.2040 & 3.9761 & 4.20888 & 4.00739 & 4.67196 \\
\hline & 1 & 3.3664 & 4.1531 & 3.36677 & 4.15802 & 3.40372 & 4.62619 \\
\hline & 2 & 2.97197 & 4.1039 & 2.9724 & 4.1087 & 3.01418 & 4.58214 \\
\hline \multirow{3}{*}{2} & 0 & 13.8613 & 16.6174 & 13.8617 & 16.6223 & 13.8961 & 17.1070 \\
\hline & 1 & 8.63113 & 15.8535 & 8.6317 & 15.8586 & 8.6868 & 16.3659 \\
\hline & 2 & 6.7975 & 15.1861 & 6.7982 & 15.1914 & 6.8680 & 15.7203 \\
\hline \multirow{3}{*}{3} & 0 & 26.6439 & 36.6870 & 26.6443 & 36.692 & 26.6834 & 37.1852 \\
\hline & 1 & 12.4867 & 33.1868 & 12.4875 & 33.1923 & 12.5707 & 33.7367 \\
\hline & 2 & 9.3582 & 30.5288 & 9.3593 & 30.5347 & 9.4708 & 31.1257 \\
\hline \multirow{3}{*}{4} & 0 & 40.6913 & 63.6119 & 40.6918 & 63.6169 & 40.7368 & 64.1183 \\
\hline & 1 & 15.0435 & 53.8623 & 15.0447 & 53.8683 & 15.1666 & 54.4595 \\
\hline & 2 & 11.0206 & 47.5515 & 11.0223 & 47.5583 & 11.1872 & 48.2269 \\
\hline \multirow{3}{*}{5} & 0 & 55.3223 & 96.4726 & 53.3228 & 96.4777 & 55.3745 & 96.9885 \\
\hline & 1 & 16.7801 & 75.8698 & 16.7818 & 75.8763 & 16.9512 & 76.5248 \\
\hline & 2 & 12.1480 & 64.5493 & 12.1504 & 64.557 & 12.3832 & 65.3179 \\
\hline
\end{tabular}

Table 7. Dimensionless frequency $\bar{\omega}$ of FG nanobeam with respect to increasing Winkler coefficient, mode number, and nonlocal parameter.

\begin{tabular}{|c|c|c|c|c|c|c|c|}
\hline \multirow{3}{*}{$n$} & \multirow{3}{*}{$\mu\left(\mathrm{nm}^{2}\right)$} & \multicolumn{6}{|c|}{$C_{w}\left(\mathrm{nN} / \mathrm{nm}^{2}\right)$} \\
\hline & & \multicolumn{2}{|c|}{$10^{4}$} & \multicolumn{2}{|c|}{$10^{6}$} & \multicolumn{2}{|c|}{$10^{8}$} \\
\hline & & $l / h=5$ & $l / h=20$ & $l / h=5$ & $l / h=20$ & $l / h=5$ & $l / h=20$ \\
\hline \multirow{3}{*}{1} & 0 & 3.9757 & 4.2079 & 3.9773 & 4.5870 & 4.1336 & 18.8251 \\
\hline & 1 & 3.3664 & 4.1950 & 3.7947 & 4.5751 & 3.9581 & 18.8222 \\
\hline & 2 & 2.9720 & 4.1059 & 2.9730 & 4.3041 & 3.0778 & 13.6087 \\
\hline \multirow{3}{*}{2} & 0 & 13.8613 & 16.6183 & 13.8618 & 16.7177 & 13.9053 & 24.7136 \\
\hline & 1 & 11.7368 & 16.4171 & 11.7374 & 16.5177 & 11.7887 & 24.5787 \\
\hline & 2 & 6.7975 & 15.1866 & 6.7979 & 15.2410 & 6.84225 & 19.9481 \\
\hline \multirow{3}{*}{3} & 0 & 26.6439 & 36.6874 & 26.6441 & 36.7321 & 26.6661 & 40.9564 \\
\hline & 1 & 19.3895 & 35.7096 & 19.3898 & 35.7555 & 19.4201 & 40.0829 \\
\hline & 2 & 9.3582 & 30.5290 & 9.3585 & 30.5558 & 9.3898 & 33.1323 \\
\hline \multirow{3}{*}{4} & 0 & 40.6913 & 63.6121 & 40.6915 & 63.6376 & 40.7057 & 66.1376 \\
\hline & 1 & 25.3375 & 60.6878 & 25.3378 & 60.7145 & 25.3607 & 63.3300 \\
\hline & 2 & 11.0206 & 47.5516 & 11.0208 & 47.5687 & 11.0471 & 49.2443 \\
\hline \multirow{3}{*}{5} & 0 & 55.3223 & 96.4727 & 55.3224 & 96.4893 & 55.3329 & 98.1357 \\
\hline & 1 & 29.7097 & 89.797 & 29.7099 & 89.8148 & 29.7294 & 91.5813 \\
\hline & 2 & 12.1480 & 64.5494 & 12.1482 & 64.5618 & 12.1720 & 65.7909 \\
\hline
\end{tabular}

difference percentage (FDPBHC) which is equal to:

$$
\frac{\mathrm{CBT}-\mathrm{HSDTs}}{\mathrm{HSDTs}} \times 100 .
$$

CBT overestimates the fundamental frequency and critical buckling load; in addition, it underestimates the bending deflection of a beam $[59,60]$. So, HSDTs can predict these parameters more precisely as compared to the classic beam theory by considering the effect of transverse shear strain on the thickness of a beam.

Figure 2 depicts $L / h=10$ and $p=0.1$. It can be seen that TBT, HBT, and HSDT1 have identically 
Table 8. Dimensionless buckling loads $\bar{N}$ of FG nanobeam with respect to increasing Pasternak coefficient, mode number, and nonlocal parameter.

\begin{tabular}{|c|c|c|c|c|c|c|c|}
\hline \multirow{3}{*}{$n$} & \multirow{3}{*}{$\mu\left(\mathrm{nm}^{2}\right)$} & \multicolumn{6}{|c|}{$C_{p}(\mathrm{nN})$} \\
\hline & & \multicolumn{2}{|c|}{$10^{4}$} & \multicolumn{2}{|c|}{$10^{6}$} & \multicolumn{2}{|c|}{$10^{8}$} \\
\hline & & $l / h=5$ & $l / h=20$ & $l / h=5$ & $l / h=20$ & $l / h=5$ & $l / h=20$ \\
\hline \multirow{3}{*}{1} & 0 & 4.4655 & 4.9135 & 4.4647 & 4.9021 & 4.3936 & 3.7641 \\
\hline & 1 & 3.2015 & 4.7952 & 3.2008 & 4.7838 & 3.1297 & 3.6458 \\
\hline & 2 & 2.4953 & 4.6824 & 2.4946 & 4.6710 & 2.4234 & 3.5330 \\
\hline \multirow{3}{*}{2} & 0 & 8.7361 & 19.4865 & 8.7354 & 19.4751 & 8.6642 & 16.3372 \\
\hline & 1 & 3.3872 & 17.7360 & 3.3865 & 17.7247 & 3.3154 & 16.5867 \\
\hline & 2 & 2.1009 & 16.2741 & 2.1002 & 16.2627 & 2.02905 & 15.1248 \\
\hline \multirow{3}{*}{3} & 0 & 6.5737 & 42.6713 & 6.57295 & 42.6599 & 6.5018 & 41.5219 \\
\hline & 1 & 1.4438 & 34.9173 & 1.44307 & 34.9059 & 1.3719 & 33.7680 \\
\hline & 2 & 0.8109 & 29.5480 & 0.8102 & 29.5366 & 0.7391 & 28.3987 \\
\hline \multirow{3}{*}{4} & 0 & 4.3467 & 71.4473 & 4.34599 & 71.4359 & 4.27487 & 70.2980 \\
\hline & 1 & 0.59409 & 51.2246 & 0.59337 & 51.2132 & 0.5222 & 50.0752 \\
\hline & 2 & 0.3188 & 39.9243 & 0.3181 & 39.9129 & 0.2470 & 38.7749 \\
\hline \multirow{3}{*}{5} & 0 & 2.9944 & 100.4270 & 2.9937 & 100.4150 & 2.92259 & 99.2774 \\
\hline & 1 & 0.2755 & 62.1125 & 0.2748 & 62.1011 & 0.2036 & 60.9632 \\
\hline & 2 & 0.1444 & 44.9597 & 0.1437 & 44.9483 & 0.0725 & 43.8104 \\
\hline
\end{tabular}

Table 9. Dimensionless buckling loads $\bar{N}$ of FG nanobeam with respect to increasing Winkler coefficient, mode number, and nonlocal parameter.

\begin{tabular}{|c|c|c|c|c|c|c|c|}
\hline \multirow{3}{*}{$n$} & \multirow{3}{*}{$\mu\left(\mathrm{nm}^{2}\right)$} & \multicolumn{6}{|c|}{$C_{w}\left(\mathrm{nN} / \mathrm{nm}^{2}\right)$} \\
\hline & & \multicolumn{2}{|c|}{$10^{4}$} & \multicolumn{2}{|c|}{$10^{6}$} & \multicolumn{2}{|c|}{$10^{8}$} \\
\hline & & $l / h=5$ & $l / h=20$ & $l / h=5$ & $l / h=20$ & $l / h=5$ & $l / h=20$ \\
\hline \multirow{3}{*}{1} & 0 & 4.4654 & 4.9089 & 4.4636 & 4.4477 & 4.2834 & 3.4152 \\
\hline & 1 & 3.2015 & 4.7906 & 3.1997 & 4.3294 & 3.0196 & 3.2946 \\
\hline & 2 & 2.4953 & 4.6779 & 2.4934 & 4.2167 & 2.3133 & 3.1894 \\
\hline \multirow{3}{*}{2} & 0 & 8.7361 & 19.4855 & 8.7356 & 19.3702 & 8.6906 & 7.8400 \\
\hline & 1 & 3.3872 & 17.7350 & 3.3868 & 17.6197 & 3.3417 & 6.0895 \\
\hline & 2 & 2.1009 & 16.2731 & 2.1004 & 16.1578 & 2.0554 & 4.6276 \\
\hline \multirow{3}{*}{3} & 0 & 6.5737 & 42.6709 & 6.5735 & 42.6196 & 6.5534 & 37.4951 \\
\hline & 1 & 1.4438 & 34.917 & 1.4436 & 34.8657 & 1.4235 & 29.7412 \\
\hline & 2 & 0.8109 & 29.5476 & 0.8107 & 29.4964 & 0.7907 & 24.3719 \\
\hline \multirow{3}{*}{4} & 0 & 4.34671 & 71.4471 & 4.34659 & 71.4183 & 4.3353 & 68.5358 \\
\hline & 1 & 0.5941 & 51.2244 & 0.5940 & 51.1956 & 0.5827 & 48.3131 \\
\hline & 2 & 0.3188 & 39.9241 & 0.3187 & 39.8953 & 0.3077 & 37.0127 \\
\hline \multirow{3}{*}{5} & 0 & 2.9944 & 100.4270 & 2.9944 & 100.4080 & 2.9872 & 98.5634 \\
\hline & 1 & 0.2755 & 62.1124 & 0.2754 & 62.0940 & 0.26821 & 60.2492 \\
\hline & 2 & 0.1444 & 44.9597 & 0.1443 & 44.9412 & 0.1371 & 43.0964 \\
\hline
\end{tabular}

the same results. Also, it can be considered that SBT has the same results with those of TBT, HBT, and HSDT1 for local amount $(\mu=0)$ as well; however, this result has changed for the nonlocal model by the increase in the nonlocal parameter. Moreover, $\mathrm{ABT}$ and EBT give the same results for fundamental frequency. So, one of these aforementioned theories can be selected to take into account the effect of transverse shear strain on the nanobeam. Figure 3 presents the fundamental frequency differences for $L / h=10$ and $p=1$. The results presented in Figure 3 are similar to those presented in Figure 2. But, with an increase in gradient index parameter, the results for the natural frequency of local model $(\mu=0)$, obtained from the 


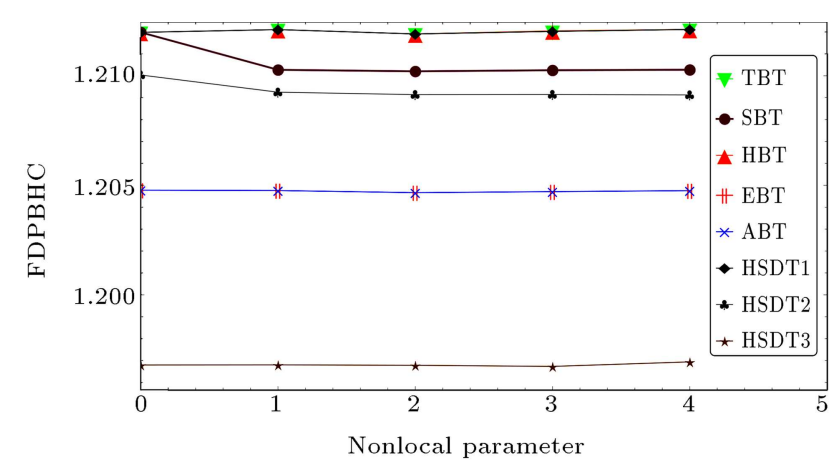

Figure 2. Fundamental frequency difference between various higher order theories and Euler-Bernoulli beam theory for $L / h=10$ and $p=0.1$.

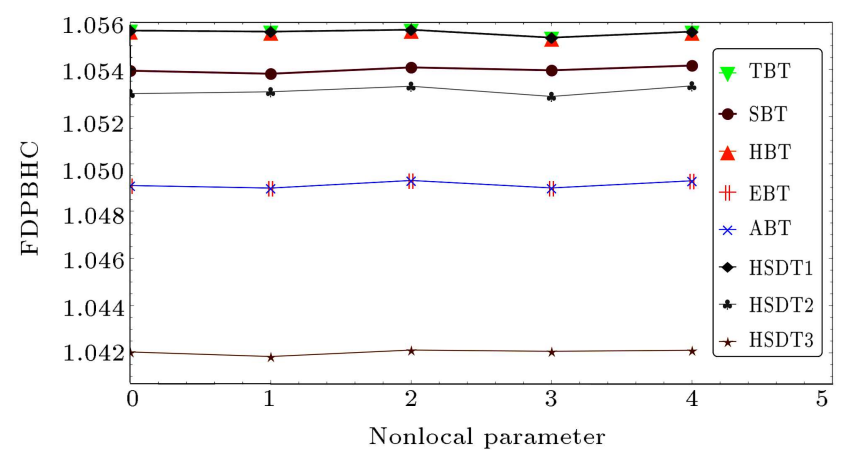

Figure 3. Fundamental frequency difference between various higher order theories and Euler-Bernoulli beam theory for $L / h=10$ and $p=1$.

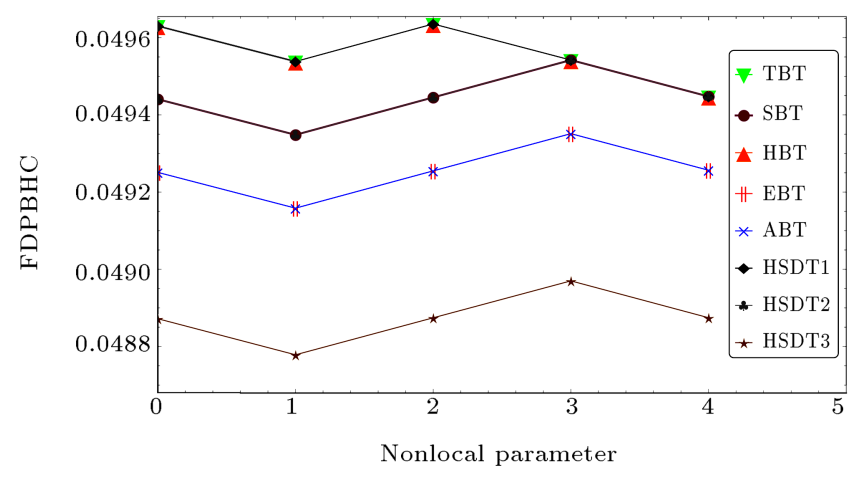

Figure 4. Fundamental frequency difference between various higher order theories and Euler-Bernoulli beam theory for $L / h=50$ and $p=0.1$.

theory of SBT, are not the same as those of TBT, HBT, and HSDT1 theories in Figure 2 (see Figure 3).

The selected parameters in Figure 4 are $L / h=50$ and $p=0.1$. As mentioned before, it is clear that the results for TBT, HBT, and HSDT1 are the same. On the other hand, the figures show the same results for SBT and HSDT2. In addition, it can be concluded for $\mu \geq 3\left(\mathrm{~nm}^{2}\right)$ that these five theories (TBT, HBT, HSDT1, SBT, and HSDT2) give the same results. Hence, one of these theories can be selected, instead of all, to consider the effect of transverse shear strain on

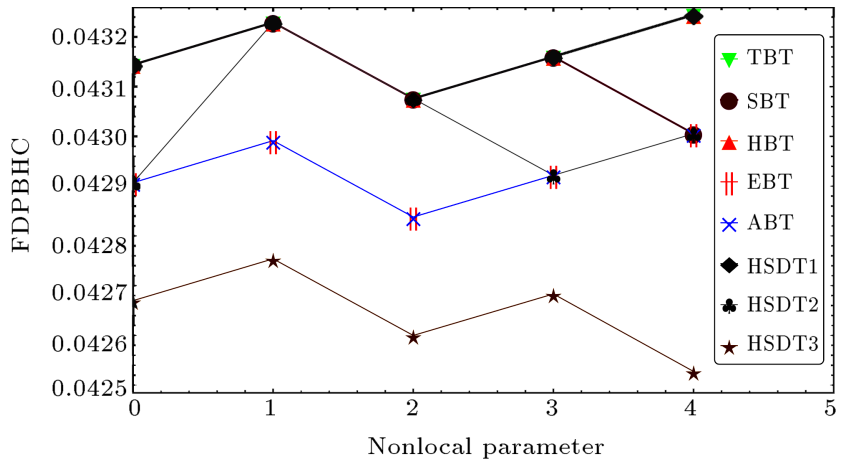

Figure 5. Fundamental frequency difference between various higher order theories and Euler-Bernoulli beam theory for $L / h=50$ and $p=1$.

the nanobeam. Figure 5 has been plotted for $L / h=50$ and $p=1$ in order to show fundamental frequency differences between HSDTs and CBT. As said before, TBT, HBT, and HSDT1 give the same results for all the values of the nonlocal parameters. It is notable that the results in SBT are the same with TBT, HBT, and HSDT1 for $0\left(\mathrm{~nm}^{2}\right) \leq \mu \leq 3\left(\mathrm{~nm}^{2}\right)$. Moreover, HSDT2 is coincided with TBT, HBT, and HSDT1 for $1\left(\mathrm{~nm}^{2}\right) \leq \mu \leq 2\left(\mathrm{~nm}^{2}\right)$; moreover, it is also coincided with ABT and EBT for $3\left(\mathrm{~nm}^{2}\right) \leq \mu \leq 4\left(\mathrm{~nm}^{2}\right)$. Finally, in evaluations of Figures 2-5, it can be seen that the HSDT3 predicts closer values to CBT in comparison with the other theories; it can be also noted that the largest differences along with CBT are in TBT, HBT, and HSDT1 theories as compared to the other theories.

Since there are no differences between the results of shear deformation beam theories, TBT is used in the rest of figures. The effects of both the gradient index and the nonlocal parameter on the dimensionless buckling load of a FG nanobeam versus a wide range of Pasternak coefficient are depicted in Figure 6. Also, the effects of length-to-thickness ratio and nonlocal parameter on the dimensionless buckling load for increasing Winkler coefficients are shown in Figure 7 . Then, in Figure 8, the effects of the gradient index and the nonlocal parameter on the dimensionless buckling load versus a wide range of Pasternak coefficient are sketched. Moreover, in Figure 9, dimensionless buckling load according to Winkler coefficient has been depicted for the variations of the nonlocal parameter and the gradient index.

Figure 6 shows the effects of the nonlocal parameter and different aspect ratios on the dimensionless deflection of FG nanobeam for the increasing Pasternak coefficients. It can be considered that the increase in Pasternak coefficient causes a decrease in the dimensionless deflection in a nonlinear way, especially for $L / h>7$. Also, with the increase in the Pasternak coefficient, especially for $C_{p}>10^{12}$, the results of the dimensionless deflection in various aspect ratios are the 


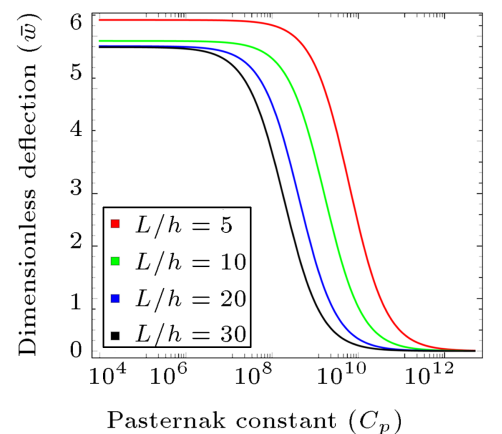

(a) $\mu=0$

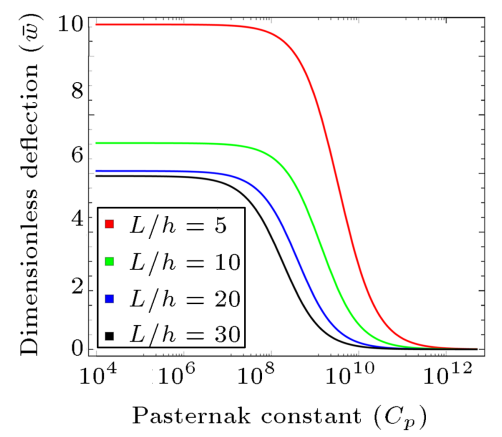

(c) $\mu=2$

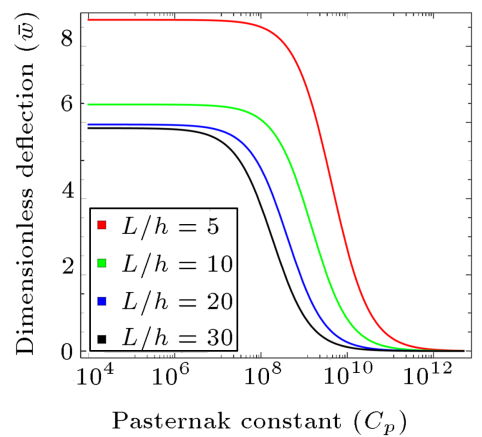

(b) $\mu=1$

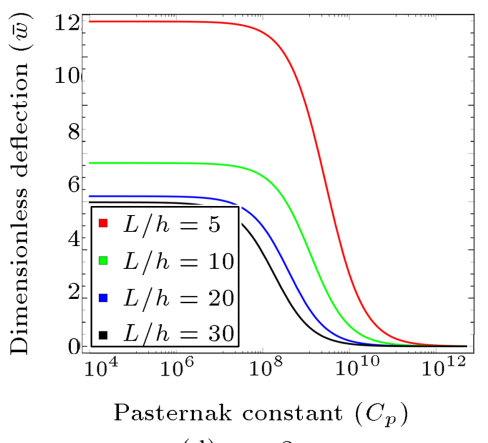

(d) $\mu=3$

Figure 6. The variation of dimensionless deflection due to increasing Pasternak elastic foundation $C_{p}\left(C_{w}=0\right)$ of FG nanobeam lying on Winkler-Pasternak elastic foundation model for various aspect ratios and $p=1$ : (a) $\mu=0$, (b) $\mu=1$, (c) $\mu=2$, and (d) $\mu=3$.

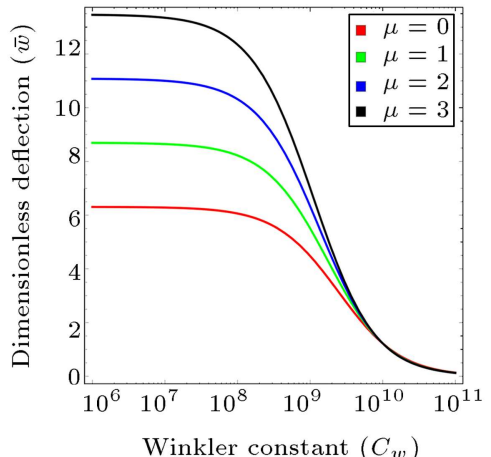

(a) $L / h=5$

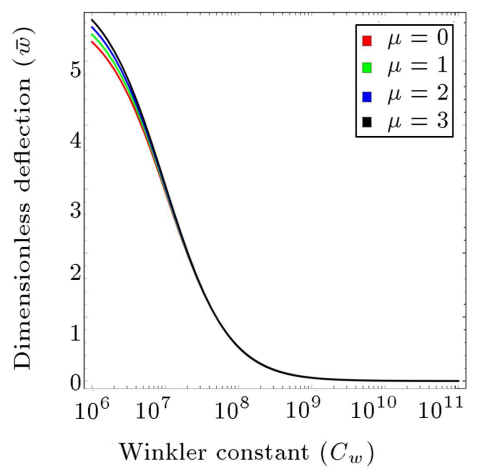

(c) $L / h=20$

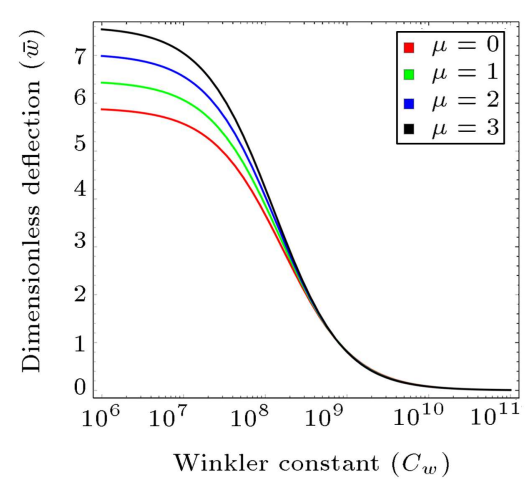

(b) $L / h=10$

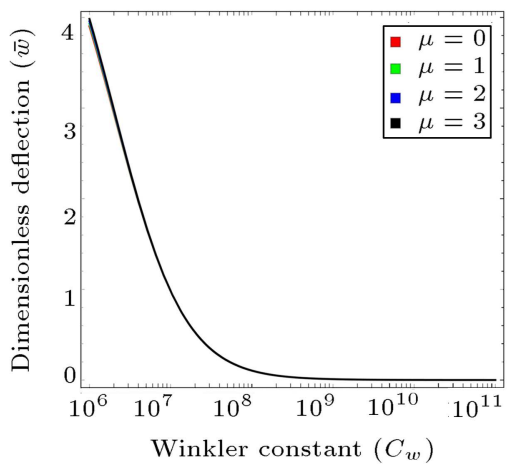

(d) $L / h=30$

Figure 7. The variation of dimensionless deflection due to increasing Winkler elastic foundation $C_{w}\left(C_{p}=0\right)$ of FG nanobeam lying on Winkler-Pasternak elastic foundation model for various nonlocal parameters and $P=1:(\mathrm{a}) P=1,(\mathrm{~b})$ $L / h=10,(\mathrm{c}) L / h=20$, and (d) $L / h=30$. 


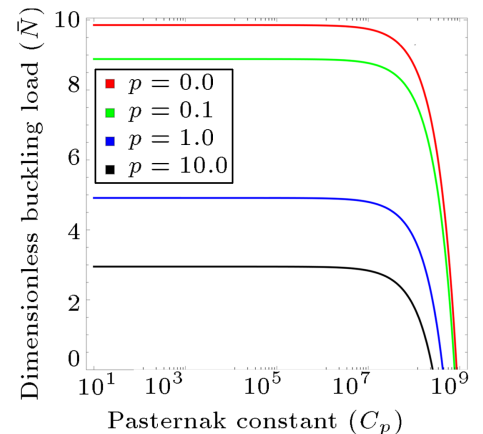

(a) $\mu=0$

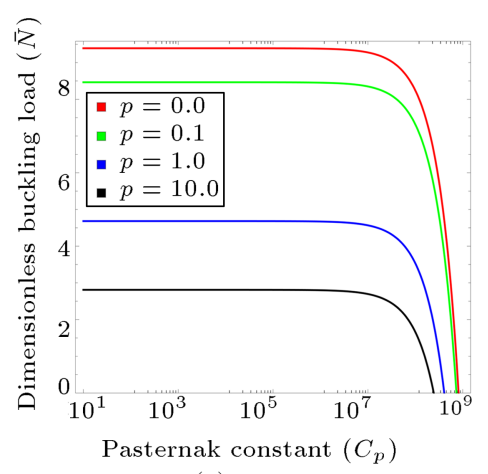

(c) $\mu=2$

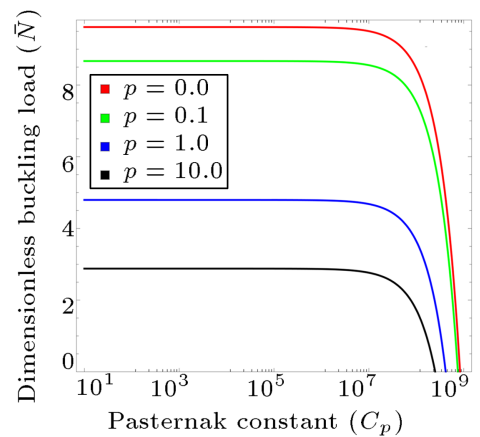

(b) $\mu=1$

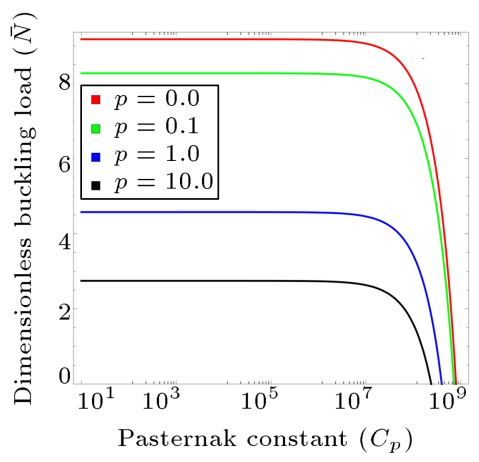

(d) $\mu=3$

Figure 8. The variation of dimensionless buckling load due to increasing Pasternak elastic coefficient $C_{p}\left(C_{w}=0\right)$ of FG nanobeam lying on Winkler-Pasternak elastic foundation model for various gradient indexes and $L / h=20$ : (a) $\mu=0,(\mathrm{~b})$ $\mu=1$, (c) $\mu=2$, and (d) $\mu=3$.

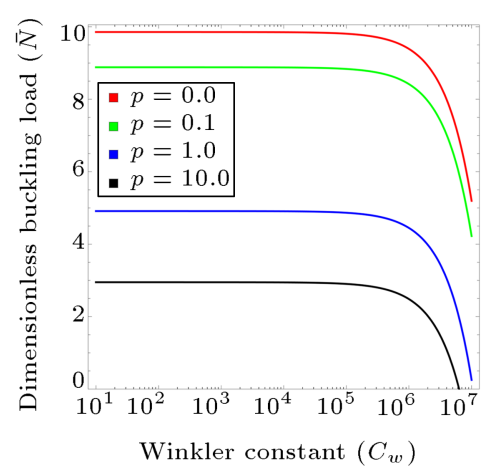

(a) $\mu=0$

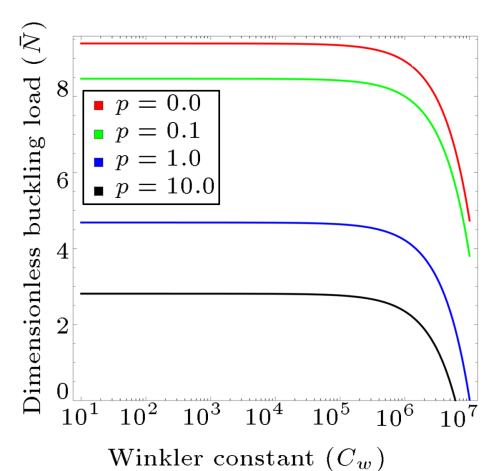

(c) $\mu=2$

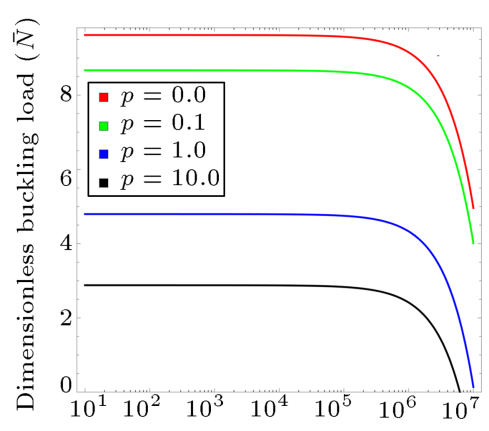

Winkler constant $\left(C_{w}\right)$

(b) $\mu=1$

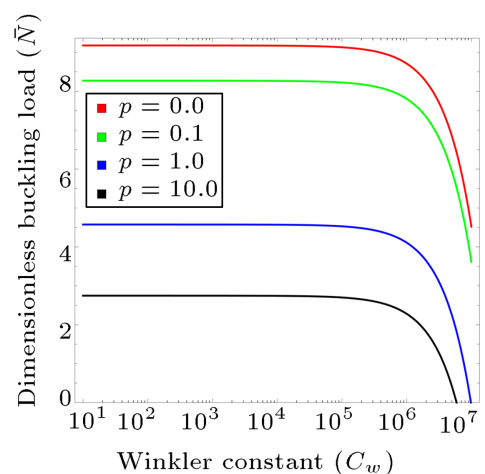

(d) $\mu=3$

Figure 9. The variation of dimensionless buckling load due to increasing Winkler elastic coefficient $C_{w}\left(C_{p}=0\right)$ of FG nanobeam lying on Winkler-Pasternak elastic foundation model for various gradient indexes and $L / h=20$ : (a) $\mu=0$, (b) $\mu=1$, (c) $\mu=2$, and (d) $\mu=3$. 
same. Furthermore, an increase in aspect ratio leads to a decrease in dimensionless deflection. In addition, by the increase in the nonlocal parameter, it can be seen that the results of the dimensionless deflection in various aspect ratios are the same. Finally, it can be considered that a significant increase in the dimensionless deflection will not be affected by an increase in the nonlocal parameter. So, it can be concluded that the influence of Pasternak elastic foundation on dimensionless deflection of nanobeam becomes more prominent as compared to the nonlocal effect.

Figure 7 is proposed in order to illustrate the effect of the Winkler coefficient on the dimensionless deflection for the different nonlocal parameter and aspect ratios. It can be seen that the dimensionless deflection reduces by the increase in the Winkler coefficient, especially for the higher value of this parameter $\left(C w>10^{8}\right)$. Also, it can be noted that by the increase in the aspect ratio, the dimensionless deflection reduces in all cases. Furthermore, when the nonlocal parameter reduces, the dimensionless deflection of nanobeam reduces as well. Moreover, it can be seen that the effect of nonlocal parameter on the nanobeam has been diminished by the increase in aspect ratio values. So, this shows that the effect of Winkler elastic foundation on dimensionless deflection of slender nanobeam (in higher value of the aspect ratio) becomes more prominent as compared to the nonlocal effect. On the other hand, the effect of Winkler coefficient on the reduction of nanobeam deflection is more obvious in the higher values of the aspect ratio. Hence, The Winkler foundation can be used to decrease the deflection of slender nanobeam.

There are two curves which have been plotted for understanding the effect of different nonlocal parameters and different gradient indexes on the variation of critical buckling loads of FG nanobeam embedded in the Winkler-Pasternak foundations. Figures 8 and 9 show the variation of critical buckling load with respect to Pasternak and Winkler coefficients, respectively. It should be noted that for the higher values of the Pasternak and Winkler coefficients, the critical buckling load of nanobeam is reduced, especially for $C_{w}, C_{p}>10^{6}$. This is due to the higher stiffness amounts of the elastic foundation which leads to the reduction of critical buckling load. Also, it can be noted that with the increase in the gradient index, the dimensionless buckling load decreases. So, it can be seen that the FG nanobeam will be softer for the higher values of the gradient index. For higher value of nonlocal parameter $\mu$, the critical buckling loads of FG nanobeam with elastic foundation model reduce. In addition, Figures 8 and 9 illustrate that the nonlocal parameter, will not have significant effect on the buckling load resting on the elastic foundation. This shows that the effect of stiffness of elastic foundation becomes more significant on the buckling load of the nanobeam as compared to that of nonlocal parameter.

\section{Conclusion}

In the present study, the exact solution was obtained for bending, buckling, and free vibration of the FG nanobeam lying on elastic foundation under axial and distributed transverse loads. Different HSDTs and the nonlocal beam theory of Eringen have been used to take into account both the effects of shear strain and nanoscale. The main results obtained from this study are as follows:

The present paper demonstrates that the bending deflection, static buckling, and free vibration behavior of nanobeam are strongly dependent on the Winkler and Pasternak elastic foundation, aspect ratio, gradient index, and nonlocal parameter.

The effect of the nonlocal parameter on the fundamental frequency of the FG nanobeam was reduced by the increase in the aspect ratio parameter, especially for the higher value of the elastic foundation coefficients. Also, it can be seen that the effect of the Winkler-Pasternak coefficients on the fundamental frequency of the nanobeam can be neglected for the lower value of these elastic coefficients. Moreover, it can be declared that the increase in dimensionless frequency depends on the increase in foundation parameters, especially for higher mode numbers. Further, it was shown that TBT, HBT, and HSDT1 have the same results in order to derive fundamental frequency of the nanobeam (ABT and EBT are the same as well) in all of the different selected aspect ratios and gradient index parameters. Besides, other theories give the same results in some cases of aspect ratios and gradient indexes. Moreover, it can be concluded that the influence of Winkler-Pasternak elastic foundation on dimensionless deflection and critical buckling load of nanobeam, especially in the higher value of the aspect ratio, becomes more significant as compared to the nonlocal effect. In addition, it is noteworthy to mention that the effect of mode number on the increase in the buckling loads has different behaviors regarding thin and thick nanobeam types. Furthermore, it should be noted that for the higher values of the Pasternak and Winkler coefficients, the dimensionless deflection and critical buckling load of nanobeam are reduced. In brief, considering these effects can be useful to investigate the nonlocal bending, buckling, and vibration of nano-devices as a smart controller in reality.

\section{References}

1. Sun, C. and Zhang, H. "Size-dependent elastic moduli of platelike nanomaterials", Journal of Applied Physics., 93(2), pp. 1212-1218 (2003). 
2. Eringen, A.C. and Edelen, D.G.B. "On nonlocal elasticity", International Journal of Engineering Science, 10(3), pp. 233-248 (1972).

3. Eringen, A.C. "On differential equations of nonlocal elasticity and solutions of screw dislocation and surface waves", Journal of Applied Physics, 54(9), pp. 47034710 (1983).

4. Eringen, A.C., Nonlocal Continuum Field Theories, Springer Science \& Business Media (2002).

5. Reddy, J. "Nonlocal theories for bending, buckling and vibration of beams", International Journal of Engineering Science, 45(2), pp. 288-307 (2007).

6. Rahmani, O., Asemani, S.S. and Hosseini, S.A.H. "Study the buckling of functionally graded nanobeams in elastic medium with surface effects based on a nonlocal theory", Journal of Computational and Theoretical Nanoscience, 12(10), pp. 3162-3170 (2015).

7. Civalek, Ö. and Akgöz, B. "Static analysis of single walled carbon nanotubes (SWCNT) based on Eringen's nonlocal elasticity theory", International Journal of Engineering and Applied Sciences, 1(2) pp. 47-56 (2009).

8. Hosseini, S.A.H. and Rahmani, O. "Exact solution for axial and transverse dynamic response of functionally graded nanobeam under moving constant load based on nonlocal elasticity theory", Meccanica, 52, pp. 1441-1457 (2017).

9. Şimşek, M. and Yurtcu, H. "Analytical solutions for bending and buckling of functionally graded nanobeams based on the nonlocal Timoshenko beam theory", Composite Structures, 97, pp. 378-386 (2013).

10. Uymaz, B. "Forced vibration analysis of functionally graded beams using nonlocal elasticity", Composite Structures, 105, pp. 227-239 (2013).

11. Hosseini, S.A.H. and Rahmani, O. "Thermomechanical vibration of curved functionally graded nanobeam based on nonlocal elasticity", Journal of Thermal Stresses, 39(10), pp. 1252-1267 (2016).

12. Hosseini, S. and Rahmani, O. "Surface effects on buckling of double nanobeam system based on nonlocal Timoshenko model", International Journal of Structural Stability and Dynamics, 16(10), pp. 122-169 (2016).

13. Hosseini, S. and Rahmani, O. "Free vibration of shallow and deep curved FG nanobeam via nonlocal Timoshenko curved beam model", Applied Physics A, 122(3), pp. 1-11 (2016).

14. Demir, Ç. and Civalek, Ö. "Torsional and longitudinal frequency and wave response of microtubules based on the nonlocal continuum and nonlocal discrete models", Applied Mathematical Modelling, 37(22), pp. 93559367 (2013).

15. Jia, X., Yang, J., Kitipornchai, S. and Lim, C. "Forced vibration of electrically actuated FGM microswitches", Procedia Engineering, 14, pp. 280-287 (2011).
16. Shariat, B., Liu, Y., Meng, Q. and Rio, G. "Analytical modelling of functionally graded NiTi shape memory alloy plates under tensile loading and recovery of deformation upon heating", Acta Materialia, 61(9), pp. 3411-3421 (2013).

17. Carbonari, R.C., Silva, E.C. and Paulino, G.H. "Multiactuated functionally graded piezoelectric micro-tools design: A multiphysics topology optimization approach", International Journal for Numerical Methods in Engineering, 77(3), pp. 301-336 (2009).

18. Mohammadi-Alasti, B., Rezazadeh, G., Borgheei, A.M., Minaei, S. and Habibifar, R. "On the mechanical behavior of a functionally graded micro-beam subjected to a thermal moment and nonlinear electrostatic pressure", Composite Structures, 93(6), pp. 1516-1525 (2011).

19. Zhang, J. and Fu, Y. "Pull-in analysis of electrically actuated viscoelastic microbeams based on a modified couple stress theory", Meccanica, 47(7), pp. 1649-1658 (2012).

20. Meng, Q., Liu, Y., Yang, H., Shariat, B.S. and Nam, T.-h. "Functionally graded NiTi strips prepared by laser surface anneal", Acta Materialia, 60(4), pp. 16581668 (2012).

21. Shariat, B.S., Liu, Y. and Rio, G. "Finite element computational modelling and experimental investigation of perforated NiTi plates under tension", Materials Research Bulletin, 48(12), pp. 5099-5104 (2013).

22. Simsek, M. and Kocaturk, T. "Free and forced vibration of a functionally graded beam subjected to a concentrated moving harmonic load", Compos Struct, 90(4), pp. 465-73 (2009).

23. Yoon, J., Ru, C. and Mioduchowski, A. "Vibration of an embedded multiwall carbon nanotube", Composites Science and Technology, 63(11), pp. 1533-1542 (2003).

24. Chen, W., Lü, C. and Bian, Z. "A mixed method for bending and free vibration of beams resting on a Pasternak elastic foundation", Applied Mathematical Modelling, 28(10), pp. 877-890 (2004).

25. Ying, J., Lü, C. and Chen, W. "Two-dimensional elasticity solutions for functionally graded beams resting on elastic foundations", Composite Structures, 84(3), pp. 209-219 (2008).

26. Murmu, T. and Pradhan, S. "Thermo-mechanical vibration of a single-walled carbon nanotube embedded in an elastic medium based on nonlocal elasticity theory", Computational Materials Science, 46(4), pp. 854-859 (2009).

27. Soltani, P., Bahar, P. and Farshidianfar, A. "An efficient GDQ model for vibration analysis of a multiwall carbon nanotube on Pasternak foundation with general boundary conditions", Proceedings of the Institution of Mechanical Engineers, Part C: Journal of Mechanical Engineering Science, 225(7), pp. 1730-1741 (2011).

28. Niknam, H. and Aghdam, M. "A semi analytical approach for large amplitude free vibration and buckling of nonlocal FG beams resting on elastic foundation", Composite Structures, 119, pp. 452-462 (2015). 
29. Civalek, Ö. and Demir, C. "A simple mathematical model of microtubules surrounded by an elastic matrix by nonlocal finite element method", Applied Mathematics and Computation, 289, pp. 335-352 (2016).

30. Thai, H.-T. and Vo, T.P. "Bending and free vibration of functionally graded beams using various higherorder shear deformation beam theories", International Journal of Mechanical Sciences, 62(1), pp. 57-66 (2012).

31. Yang, J. and Chen, Y. "Free vibration and buckling analyses of functionally graded beams with edge cracks", Compos Struct., 83(1), pp. 48-60 (2008).

32. Alshorbagy, A., Eltaher, M. and Mahmoud, F. "Free vibration characteristics of a functionally graded beam by finite element method", Appl. Math. Modell., 35(1), pp. 412-25 (2011).

33. Chakraborty, A., Gopalakrishnan, S. and N, R.J. "A new beam finite element for the analysis of functionally graded materials", Int. J. Mech. Sci., 45(3), pp. 51939. (2003).

34. XF, L. "A unified approach for analyzing static and dynamic behaviors of functionally graded Timoshenko and Euler-Bernoulli beams", J. Sound Vib., 318(4-5), pp. 1210-1229 (2008).

35. Sina, S.A., Navazi, H.M. and Haddadpour, H. "An analytical method for free vibration analysis of functionally graded beams.", Materials \& Design, 30(3), pp. 741-747 (2009).

36. Wei, D., Liu, Y. and Z., X. "An analytical method for free vibration analysis of functionally graded beams with edge cracks.", J. Sound Vib., 331(7), pp. 1686700. (2012).

37. Aydogdu, M. and Taskin, V. "Free vibration analysis of functionally graded beams with simply supported edges", Materials \& Design, 28(5), pp. 1651-1656 (2007).

38. Kadoli, R., Akhtar, K. and Ganesan, N. "Static analysis of functionally graded beams using higher order shear deformation theory", Applied Mathematical Modelling, 32(12), pp. 2509-2525 (2008).

39. Şimşek, M. "Static analysis of a functionally graded beam under a uniformly distributed load by Ritz method", Int. J. Eng. Appl. Sci., 1(3), pp. 1-11 (2009).

40. Giunta, G., Belouettar, S. and Carrera, E. "Analysis of FGM beams by means of classical and advanced theories", Mechanics of Advanced Materials and Structures, $\mathbf{1 7}(8)$, pp. 622-635 (2010).

41. Giunta, G., Belouettar, S. and Carrera, E. "Analysis of FGM beams by means of a unified formulation", In IOP Conference Series: Materials Science and Engineering, IOP Publishing (2010).

42. Giunta, G., Crisafulli, D., Belouettar, S. and Carrera, E. "Hierarchical theories for the free vibration analysis of functionally graded beams", Composite Structures, 94(1), pp. 68-74 (2011).
43. Lei, J., He, Y., Zhang, B., Gan, Z. and Zeng, P. "Bending and vibration of functionally graded sinusoidal microbeams based on the strain gradient elasticity theory", International Journal of Engineering Science, 72, pp. 36-52 (2013).

44. Eltaher, M.A., Emam, S.A. and Mahmoud, F.F. "Static and stability analysis of nonlocal functionally graded nanobeams", Composite Structures, 96, pp. 8288 (2013).

45. Rahmani, O. and Pedram, O. "Analysis and modeling the size effect on vibration of functionally graded nanobeams based on nonlocal Timoshenko beam theory", International Journal of Engineering Science, 77(0), pp. 55-70 (2014).

46. Refaeinejad, V., Rahmani, O. and Hosseini, S.A.H. "Evaluation of nonlocal higher order shear deformation models for the vibrational analysis of functionally graded nanostructures", Mechanics of Advanced Materials and Structures, pp. 00-00 (2016).

47. Reddy, J.N. "A simple higher-order theory for laminated composite plates", Journal of Applied Mechanics, 51(4), pp. 745-752 (1984).

48. Touratier, M. "An efficient standard plate theory", International Journal of Engineering Science, 29(8), pp. 901-916 (1991).

49. Soldatos, K. "A transverse shear deformation theory for homogeneous monoclinic plates", Acta Mechanica., 94(3-4), pp. 195-220 (1992).

50. Karama, M., Afaq, K. and Mistou, S. "Mechanical behaviour of laminated composite beam by the new multi-layered laminated composite structures model with transverse shear stress continuity", International Journal of Solids and Structures, 40(6), pp. 1525-1546 (2003).

51. Mantari, J. and Guedes Soares, C. "Bending analysis of thick exponentially graded plates using a new trigonometric higher order shear deformation theory", Composite Structures, 94(6), pp. 1991-2000 (2012).

52. Mantari, J., Oktem, A. and Guedes Soares, C. "Bending response of functionally graded plates by using a new higher order shear deformation theory", Composite Structures, 94(2), pp. 714-723 (2012).

53. Mantari, J.L., Bonilla, E.M. and Soares, C.G "A new tangential-exponential higher order shear deformation theory for advanced composite plates", Composites Part B: Engineering, 60, pp. 319-328 (2014).

54. Scott, R.F., Foundation Analysis, Prentice-Hall London (1981).

55. Pasternak, P., On a New Method of Analysis of an Elastic Foundation by Means of Two Foundation constants, Gosudarstvennoe Izdatelstvo Literaturi po Stroitelstvu i Arkhitekture, Moscow (1954).

56. Ugural, A.C. and Fenster, S.K., Advanced Strength and Applied Elasticity, Pearson Education (2003).

57. Khajeansari, A., Baradaran, G. and Yvonnet, J. "An explicit solution for bending of nanowires lying on 
Winkler-Pasternak elastic substrate medium based on the Euler-Bernoulli beam theory", International Journal of Engineering Science, 52, pp. 115-128 (2012).

58. Şimşek, M. "Fundamental frequency analysis of functionally graded beams by using different higher-order beam theories", Nuclear Engineering and Design, 240(4), pp. 697-705 (2010).

59. Thai, H.-T. "A nonlocal beam theory for bending, buckling, and vibration of nanobeams", International Journal of Engineering Science, 52, pp. 56-64 (2012).

60. Thai, H.-T. and Vo, T.P. "A nonlocal sinusoidal shear deformation beam theory with application to bending, buckling, and vibration of nanobeams", International Journal of Engineering Science, 54, pp. 58-66 (2012).

\section{Appendix}

The expressions of $S_{i j}$ and $M_{i j}$ are given as follows:

$$
\begin{aligned}
& s_{11}=A \alpha^{2}, \quad s_{12}=-B \alpha^{3}, \quad s_{13}=-B_{s} \alpha^{3}, \\
& s_{22}=D \alpha^{4}-\bar{N} \alpha^{2}\left(1+\mu \alpha^{2}\right)+\left(C_{w}+C_{p} \alpha^{2}\right)\left(1+\mu \alpha^{2}\right), \\
& s_{23}=D_{s} \alpha^{4}-\bar{N} \alpha^{2}\left(1+\mu \alpha^{2}\right)+\left(C_{w}+C_{p} \alpha^{2}\right)\left(1+\mu \alpha^{2}\right), \\
& s_{33}=H_{s} \alpha^{4}+A_{s} \alpha^{2}-\bar{N} \alpha^{2}\left(1+\mu \alpha^{2}\right) \\
& \quad+\left(C_{w}+C_{p} \alpha^{2}\right)\left(1+\mu \alpha^{2}\right), \\
& m_{11}=I_{0}, \quad m_{12}=-I_{1} \alpha, \\
& m_{13}=-J_{1} \alpha, \quad m_{22}=I_{0}+I_{2} \alpha^{2}, \\
& m_{23}=I_{0}+J_{2} \alpha^{2}, \quad m_{33}=I_{0}+K_{2} \alpha^{2} .
\end{aligned}
$$

\section{Biographies}

Vahid Refaeinejad received his MSc degree in Mechanical Engineering from University of Zanjan, Zanjan, Iran 2015. He worked on "analysis of vibration, bending, and buckling of functionally graded nanobeam lying on Winkler and Pasternak elastic foundations based on non-local higher order deformation theory" as his master thesis. He presented some parts of his thesis in several international journal papers and conference. His research interests are analyses of stability and vibration behavior of nanostructures, smart and advanced materials, and composite structures.

Omid Rahmani is an Associated Professor and Director of "Smart Material and New Advanced Materials Laboratory", who specializes in smart composite material and nano mechanics. He has over 60 papers in the well-known journals and international conferences since 2008. Dr. Rahmani also was staying at Aalborg University, Denmark as visiting $\mathrm{PhD}$ scholar during 2010-2011.

Seyyed Amir Hossein Hosseini received his $\mathrm{PhD}$ and MSc degrees in Mechanical Engineering from University of Zanjan. He has expertise in vibration of beam and curved structures, biosensors, biophysics, dynamics of smart piezoelectric structures, nanomechanics. He has worked on $\mathrm{PhD}$ thesis entitled "mechanical behavior of curved nanobeam based on nonlocal elasticity theory". He has published more than 25 international journal papers. His current research focuses on the vibration and stability of atomic microscopic probe in real condition and drug delivery. 